\title{
Standard versus reduced genus-one Gromov-Witten invariants
}

\author{
AleKsey Zinger
}

\begin{abstract}
We give an explicit formula for the difference between the standard and reduced genus-one Gromov-Witten invariants. Combined with previous work on geometric properties of the latter, this paper makes it possible to compute the standard genus-one GW-invariants of complete intersections. In particular, we obtain a closed formula for the genus-one GW-invariants of a Calabi-Yau projective hypersurface and verify a recent mirror symmetry prediction for a sextic fourfold as a special case.
\end{abstract}

14D20, 14N35; 53D45, 53D99

\section{Introduction}

Gromov-Witten invariants are counts of holomorphic curves in symplectic manifolds that play prominent roles in string theory, symplectic topology, and algebraic geometry. A variety of predictions concerning these invariants have arisen from string theory, only some of which have been verified mathematically. These invariants are generally difficult to compute, especially in positive genera. For example, the 1991 Candelas-de la Ossa-Green-Parkes prediction [2] for the genus-zero GW-invariants of the quintic threefold was mathematically confirmed in the mid-1990s, while even low-degree cases of the 1993 Bershadsky-Cecotti-Ooguri-Vafa prediction [1] for its genus-one GW-invariants remained unaccessible until early this century.

In contrast to the genus-zero case, the expected hyperplane (or hyperplane-section) relation between $\mathrm{GW}$-invariants of a complete intersection and those of the ambient space does not hold in positive genera; see Subsection 1.2 in [16] for more details. This issue is entirely avoided by Gathmann [4] and Maulik-Pandharipande [10] by approaching GW-invariants of complete intersections through degeneration techniques. The methods of [4] and [10] can be used for low-degree checks of [1], but they do not seem to provide a ready platform for an application of combinatorial techniques as in the genus-zero case. In contrast, the failure of the expected hyperplane relation for genus-one invariants is addressed by $\mathrm{Li}$ and the author in [8] by showing that the reduced genus-one GW-invariants, defined by the author in [15], do satisfy the 
hyperplane relation. Combined with the desingularization construction of Vakil and the author [13], this last approach provides a platform suitable for an application of combinatorial techniques (the classical localization theorem); the author has used it to obtain a closed formula for the reduced GW-invariants of a Calabi-Yau projective hypersurface [16]. For symplectic manifolds of real dimension six, the standard and reduced genus-one GW-invariants without descendants differ by a multiple of the genus-zero GW-invariant. ${ }^{1}$ The prediction of [1] is thus fully verified in [16] as a special case.

Remark The ranges of applicability of the two degeneration methods and of the reduced-invariants method are very different, with a relatively limited overlap. The wider-ranging degeneration method, that of [10], can in principle be used to compute arbitrary-genus GW-invariants of low-degree low-dimension complete intersections. While the computation in each case is generally difficult, Maulik and Pandharipande have used it to compute genus-one and genus-two GW-invariants of the Enriques Calabi-Yau threefold [9]. On the other hand, the reduced-invariants method applies to arbitrary complete intersections, but at this point in the genus-one case only.

In theory, reduced genus-one GW-invariants are not new invariants, as by Proposition 3.1 in [15] the difference between these invariants and the standard ones is some combination of genus-zero GW-invariants. However, this is not part of the definition of reduced genus-one invariants and the relation described by Proposition 3.1 in [15] is not convenient for immediate applications; see Proposition 3.2 below. In this paper, we determine an explicit relation; see Theorems 1A and 1B. Combining this relation with the closed formula for the reduced genus-one GW-invariants of a CalabiYau hypersurface derived in [16], we then obtain a closed formula for the standard genus-one GW-invariants of a Calabi-Yau hypersurface; see Theorem 2. The KlemmPandharipande mirror symmetry prediction [6] for a sextic fourfold is confirmed by the $n=6$ case of Theorem 2. The $n>6$ cases of Theorem 2 go beyond even predictions, as far as the author is aware.

It is interesting to observe that only one boundary stratum of a partially regularized moduli space of genus-one stable maps accounts for the difference between the standard and reduced genus-one GW-invariants without descendants. This implies that if $X$ is a sufficiently regular almost Kahler manifold (eg a low-degree projective hypersurface), only two strata of the moduli space $\overline{\mathfrak{M}}_{1, k}(X, \beta)$ of degree- $\beta$ genus-one stable maps to $X$ with $k$ marked points contribute to the genus-one GW-invariants without descendants:

\footnotetext{
${ }^{1}$ For the purposes of this statement, all three GW-invariants are viewed as linear functionals on the same vector space (consisting of tuples of homology elements in the manifold).
} 
(i) the main stratum $\mathfrak{M}_{1, k}^{0}(X, \beta)$ consisting of stable maps from smooth domains;

(ii) the stratum $\mathfrak{M}_{1, k}^{1, \varnothing}(X, \beta)$ consisting of stable maps from a union of a smooth genus-one curve and a $\mathbb{P}^{1}$, with the map constant on the genus-one curve and with all $k$ marked points lying on the latter; see the first diagram in Figure 1.

At a first glance, this statement may seem to contradict reality, as well as Theorems $1 \mathrm{~A}$ and 1B. For example, if $n \geq 4$, the formula for the difference in Theorems $1 \mathrm{~A}$ and 1B involves the GW-invariant $\mathrm{GW}_{(2, \varnothing)}^{\beta}$ that counts two-component rational curves; this is consistent with [6]. This term may appear to arise from the stratum $\mathfrak{M}_{1, k}^{2, \varnothing}(X, \beta)$ of $\overline{\mathfrak{M}}_{1, k}(X, \beta)$ consisting of maps from a smooth genus-one curve $\mathcal{C}_{P}$ with two $\mathbb{P}^{1}$ 's attached directly to $\mathcal{C}_{P}$ so that the map is constant on $\mathcal{C}_{P}$; see the middle diagram in Figure 1. In fact, $\mathrm{GW}_{(2, \varnothing)}^{\beta}$ arises from a homology class on $\overline{\mathfrak{M}}_{0, k+1}(X, \beta)$, or equivalently from the closure of a boundary stratum of $\overline{\mathfrak{M}}_{1, k}^{1, \varnothing}(X, \beta)$. This boundary stratum lies in the intersection of $\overline{\mathfrak{M}}_{1, k}^{1, \varnothing}(X, \beta)$ with $\overline{\mathfrak{M}}_{1, k}^{2, \varnothing}(X, \beta)$; see the last diagram in Figure 1.
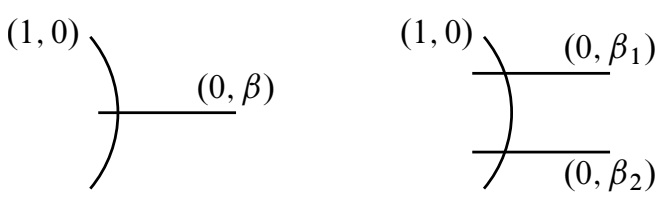

$$
\beta_{1}, \beta_{2} \neq 0 \beta_{1}+\beta_{2}=\left.\beta \quad\right|_{\left(0, \beta_{2}\right)}
$$

Figure 1: Generic elements of $\quad \overline{\mathfrak{M}}_{1, k}^{1, \varnothing}(X, \beta), \quad \overline{\mathfrak{M}}_{1, k}^{2, \varnothing}(X, \beta), \quad$ and $\overline{\mathfrak{M}}_{1, k}^{1, \varnothing}(X, \beta) \cap \overline{\mathfrak{M}}_{1, k}^{2, \varnothing}(X, \beta)$, respectively; the lines and curves represent the components of the domain of a stable map and the pair of integers next to each component indicates the genus of the component and the degree of the map on the component.

After setting up notation for GW-invariants in Section 2.1, we state the main theorem of this paper in Section 2.2. Theorem 1A expresses the difference between the standard and reduced genus-one GW-invariants as a linear combination of genus-zero invariants. The coefficients in this linear combination are top intersections of tautological classes on the blowups of moduli spaces of genus-one curves constructed in Subsection 2.3 in [13] and reviewed in Section 3.1 below. These are computable through the recursions obtained by the author in [19] and restated in Section 2.2 below; (2-9) gives an explicit formula for these coefficients when no descendants are involved. We then deduce a more compact version of Theorem 1A; Theorem 1B involves certain (un-)twisted $\psi$-classes and coefficients that satisfy simpler recursions than the coefficients in Theorem 1A. The 
descendant-free case of Theorem 1B, (2-15), is used in Section 2.3 to obtain a closed formula for the difference between the two genus-one GW-invariants of a Calabi-Yau hypersurface from a closed formula for its genus-zero $\mathrm{GW}$-invariants confirmed in the book Mirror symmetry [5]; see Lemma 2.2. Using a closed formula for the reduced genus-one GW-invariants of such a hypersurface derived in [16], we thus obtain a closed formula for its standard genus-one GW-invariants.

Theorem 1A is proved in Section 3. In addition to reviewing the blowup construction of Subsection 2.3 in [13], Section 3.1 describes natural bundle homomorphisms over moduli spaces of genus-one curves and their twisted versions. These are used to describe the difference between the two genus-one GW-invariants in Section 3.3 and to compute it explicitly in Section 3.4, respectively. The blowup construction of Subsection 2.4 in [13] for moduli spaces of genus-zero curves is used in Section 3.2 to obtain a formula for top intersections of tautological classes on blowups of moduli spaces of genus-one curves; Proposition 3.1 is used at the end of Section 3.4. Section 3.3 reviews the relevant aspects of [15], concluding with a description of the difference between the two genus-one GW-invariants; see Proposition 3.2. This difference can be computed explicitly through the direct, but rather involved, setup of [18]. Section 3.4 instead presents a more conceptual approach motivated by the blowup construction of Section 3 in [13] for moduli spaces of genus-zero maps.

The author would like to thank J Li for first drawing the author's attention to the issue of computing genus-one GW-invariants of projective hypersurfaces, R Pandharipande for pointing out the mirror symmetry prediction for a sextic fourfold in [6], and the referee for suggestions for improving the exposition. This research was partially supported by a Sloan fellowship and DMS Grant 0604874.

\section{Overview}

\subsection{Gromov-Witten invariants}

Let $(X, \omega, \mathcal{J})$ be a compact symplectic manifold with a compatible almost complex structure. If $g, k \in \overline{\mathbb{Z}}^{+}$are nonnegative integers and $\beta \in H_{2}(X ; \mathbb{Z})$, we denote by $\overline{\mathfrak{M}}_{g, k}(X, \beta ; \mathcal{J})$ the moduli space of genus- $g$ degree- $\beta \mathcal{J}$-holomorphic maps into $X$ with $k$ marked points. For each $j=1, \ldots, k$, let

$$
\mathrm{ev}_{j}: \overline{\mathfrak{M}}_{g, k}(X, \beta ; \mathcal{J}) \longrightarrow X
$$

be the evaluation map at the $j$-th marked point and let

$$
\psi_{j} \in H^{2}\left(\overline{\mathfrak{M}}_{g, k}(X, \beta ; \mathcal{J})\right)
$$

Geometry $\&$ Topology, Volume 12 (2008) 
be the first Chern class of the universal cotangent line bundle at the $j$-th marked point. More generally, if $J$ is a finite set, we denote by $\overline{\mathfrak{M}}_{0, J}(X, \beta ; \mathcal{J})$ the moduli space of genus- 0 degree- $\beta \mathcal{J}$-holomorphic maps into $X$ with marked points indexed by the set $J$ and by

$$
\mathrm{ev}_{j}: \overline{\mathfrak{M}}_{0, J}(X, \beta ; \mathcal{J}) \longrightarrow X, \quad \psi_{j} \in H^{2}\left(\overline{\mathfrak{M}}_{0, J}(X, \beta ; \mathcal{J})\right), \quad \forall j \in J,
$$

the corresponding evaluation maps and $\psi$-classes. If $\beta \neq 0$, for each $J^{\prime} \subset J$ there is a well-defined forgetful map

$$
f_{J^{\prime}}: \overline{\mathfrak{M}}_{0, J}(X, \beta ; \mathcal{J}) \longrightarrow \overline{\mathfrak{M}}_{0, J-J^{\prime}}(X, \beta ; \mathcal{J}),
$$

obtained by dropping the marked points indexed by $J^{\prime}$ from the domain of every stable map in $\overline{\mathfrak{M}}_{0, J}(X, \beta ; \mathcal{J})$ and contracting the unstable components of the resulting map.

Let

$$
\widetilde{\psi}_{j} \equiv f_{J-j}^{*} \psi_{j} \in H^{2}\left(\overline{\mathfrak{M}}_{0, J}(X, \beta ; \mathcal{J})\right)
$$

be the untwisted $j$-th $\psi$-class.

We also define moduli spaces of tuples of genus-zero stable maps. If $m \in \overline{\mathbb{Z}}^{+}$, let

$$
[m]=\left\{i \in \mathbb{Z}^{+}: 1 \leq i \leq m\right\} .
$$

If $m \in \mathbb{Z}^{+}$and $J$ is a finite set, we define

$$
\begin{aligned}
\overline{\mathfrak{M}}_{(m, J)}(X, \beta ; \mathcal{J})=\left\{\left(b_{i}\right)_{i \in[m]} \in \prod_{i=1}^{i=m} \overline{\mathfrak{M}}_{0,\{0\} \sqcup J_{i}}\left(X, \beta_{i} ; \mathcal{J}\right):\right. \\
\beta_{i} \in H_{2}(X ; \mathbb{Z})-\{0\}, J_{i} \subset J ; \sum_{i=1}^{i=m} \beta_{i}=\beta, \\
\\
\left.\bigsqcup_{i=1}^{i=m} J_{i}=J, \operatorname{ev}_{0}\left(b_{i}\right)=\operatorname{ev}_{0}\left(b_{i^{\prime}}\right) \forall i, i^{\prime} \in[m]\right\} .
\end{aligned}
$$

There is a well-defined evaluation map

$$
\mathrm{ev}_{0}: \overline{\mathfrak{M}}_{(m, J)}(X, \beta ; \mathcal{J}) \longrightarrow X, \quad\left(b_{i}\right)_{i \in[m]} \longrightarrow \operatorname{ev}_{0}\left(b_{i}\right)
$$

where $i$ is any element of $[m]$. For each $i \in[m]$, let

$$
\pi_{i}: \overline{\mathfrak{M}}_{(m, J)}(X, \beta ; \mathcal{J}) \longrightarrow \bigsqcup_{\beta_{i} \in H_{2}(X ; \mathbb{Z})} \bigsqcup_{J_{i} \subset J} \overline{\mathfrak{M}}_{0,\{0\} \sqcup J_{i}}\left(X, \beta_{i} ; \mathcal{J}\right)
$$

be the projection onto the $i$-th component. If $p \in \overline{\mathbb{Z}}^{+}$, we define

$$
\eta_{p}, \tilde{\eta}_{p} \in H^{2 p}\left(\overline{\mathfrak{M}}_{(m, J)}(X, \beta ; \mathcal{J})\right)
$$


to be the degree $-2 p$ terms of

$$
\prod_{i=1}^{i=m} \pi_{i}^{*} \frac{1}{\left(1-\psi_{0}\right)}, \prod_{i=1}^{i=m} \pi_{i}^{*} \frac{1}{\left(1-\widetilde{\psi}_{0}\right)} \in H^{*}\left(\overline{\mathfrak{M}}_{(m, J)}(X, \beta ; \mathcal{J})\right),
$$

respectively. Thus, $\eta_{p}$ and $\tilde{\eta}_{p}$ are the sums of all degree $-p$ monomials in

$$
\left\{\pi_{i}^{*} \psi_{0}: i \in[m]\right\} \quad \text { and } \quad\left\{\pi_{i}^{*} \widetilde{\psi}_{0}: i \in[m]\right\},
$$

respectively. For example, if $m=2$,

$$
\eta_{3}=\pi_{1}^{*} \psi_{0}^{3}+\pi_{2}^{*} \psi_{0}^{3}+\pi_{1}^{*} \psi_{0}^{2} \pi_{2}^{*} \psi_{0}+\pi_{1}^{*} \psi_{0} \pi_{2}^{*} \psi_{0}^{2} \in H^{6}\left(\overline{\mathfrak{M}}_{(2, J)}(X, \beta ; \mathcal{J})\right) .
$$

The symmetric group on $m$ elements, $S_{m}$, acts on $\overline{\mathfrak{M}}_{(m, J)}(X, \beta ; \mathcal{J})$ by permuting the elements of each $m$-tuple of stable maps. Let

$$
\mathcal{Z}_{(m, J)}(X, \beta ; \mathcal{J})=\overline{\mathfrak{M}}_{(m, J)}(X, \beta ; \mathcal{J}) / S_{m}
$$

Since the map ev $\mathrm{e}_{0}$ and the cohomology classes $\eta_{q}$ and $\tilde{\eta}_{q}$ on $\overline{\mathfrak{M}}_{(m, J)}(X, \beta ; \mathcal{J})$ are $S_{m}$-invariant, they descendant to the quotient:

$$
\mathrm{ev}_{0}: \mathcal{Z}_{(m, J)}(X, \beta ; \mathcal{J}) \longrightarrow X \quad \text { and } \quad \eta_{q}, \tilde{\eta}_{q} \in H^{2 q}\left(\mathcal{Z}_{(m, J)}(X, \beta ; \mathcal{J})\right)
$$

The constructions of Fukaya-Ono [3] and Li-Tian [7] endow

$$
\overline{\mathfrak{M}}_{g, k}(X, \beta ; \mathcal{J}), \quad \overline{\mathfrak{M}}_{(m, J)}(X, \beta ; \mathcal{J}), \quad \text { and } \quad \mathcal{Z}_{(m, J)}(X, \beta ; \mathcal{J})
$$

with virtual fundamental classes (VFCs). If the real dimension of $X$ is $2 n$, the first VFC is of real dimension

$$
\begin{aligned}
2 \operatorname{dim}^{\mathrm{vir}} \overline{\mathfrak{M}}_{g, k}(X, \beta ; \mathcal{J})= & 2 \operatorname{dim}_{g, k}(X, \beta) \\
& \equiv 2\left(\left\langle c_{1}(T X), \beta\right\rangle+(n-3)(1-g)+k\right) .
\end{aligned}
$$

The other two VFCs are of real dimension

$$
\begin{aligned}
2 \operatorname{dim}^{\mathrm{vir}} \overline{\mathfrak{M}}_{(m, J)}(X, \beta ; \mathcal{J}) & =2 \operatorname{dim}^{\mathrm{vir}} \mathcal{Z}_{(m, J)}(X, \beta ; \mathcal{J}) \\
& =2 \operatorname{dim}_{(m, J)}(X, \beta) \\
& \equiv 2\left(\operatorname{dim}_{0,|J|}(X, \beta)+3-2 m\right) .
\end{aligned}
$$

The VFC for $\overline{\mathfrak{M}}_{(m, J)}(X, \beta ; \mathcal{J})$ is obtained from the VFCs for $\overline{\mathfrak{M}}_{0,\{0\} \sqcup J_{i}}\left(X, \beta_{i} ; \mathcal{J}\right)$ via the usual method of a Kunneth decomposition of the (small) diagonal (eg as in the proof of commutativity of the quantum product). The $\operatorname{VFC}$ for $\mathcal{Z}_{(m, J)}(X, \beta ; \mathcal{J})$ is the homology class induced from the $S_{m}$-action on $\overline{\mathfrak{M}}_{(m, J)}(X, \beta ; \mathcal{J})$. 
For each tuple

$$
\mu \equiv\left(c_{1}, \ldots, c_{k} ; \mu_{1}, \ldots, \mu_{k}\right) \in\left(\overline{\mathbb{Z}}^{+}\right)^{k} \oplus H^{*}(X ; \mathbb{Z})^{k}
$$

such that

$$
|\mu| \equiv \sum_{j=1}^{j=k}\left(2 c_{i}+\operatorname{deg} \mu_{i}\right)=2 \operatorname{dim}_{1, k}(X, \beta),
$$

let

$$
\begin{aligned}
& \mathrm{GW}_{1, k}^{\beta}(\mu) \equiv\left\langle\left(\psi_{1}^{c_{1}} \mathrm{ev}_{1}^{*} \mu_{1}\right) \ldots\left(\psi_{k}^{c_{k}} \operatorname{ev}_{k}^{*} \mu_{k}\right),\left[\overline{\mathfrak{M}}_{1, k}(X, \beta ; \mathcal{J})\right]^{\mathrm{vir}}\right\rangle \\
& \mathrm{GW}_{1, k}^{\beta ; 0}(\mu) \equiv\left\langle\left(\psi_{1}^{c_{1}} \mathrm{ev}_{1}^{*} \mu_{1}\right) \ldots\left(\psi_{k}^{c_{k}} \operatorname{ev}_{k}^{*} \mu_{k}\right),\left[\overline{\mathfrak{M}}_{1, k}^{0}(X, \beta ; \mathcal{J})\right]^{\mathrm{vir}}\right\rangle
\end{aligned}
$$

and

be the standard and reduced genus-one degree- $\beta$ GW-invariants of $X$ corresponding to the tuple $\mu$. The latter is constructed in [15].

If $\mu$ is as in (2-3), $m \in \mathbb{Z}^{+}$, and $J \subset[k]$, let

$$
\mu_{J}=\prod_{j \in J} \mu_{j} \in H^{*}(X ; \mathbb{Z}), p_{J}(\mu)=\sum_{j \in J} c_{j}, d_{m, J}(\mu)=n-2 m-|J|+p_{J}(\mu) .
$$

If $|\mu|=2 \operatorname{dim}_{1, k}(X, \beta)$, then

$$
\operatorname{deg}\left(\left(\operatorname{ev}_{0}^{*} \mu_{J} \prod_{j \notin J}\left(\psi_{j}^{c_{j}} \operatorname{ev}_{j}^{*} \mu_{j}\right)\right) \cap\left[\mathcal{Z}_{(m,[k]-J)}(X, \beta ; \mathcal{J})\right]^{\mathrm{vir}}\right)=2 d_{m, J}(\mu)
$$

Thus, when $p+q=2 d_{m, J}(\mu), \eta \in H^{2 p}\left(\mathcal{Z}_{(m,[k]-J)}(X, \beta ; \mathcal{J})\right)$, and $\mu_{0} \in H^{2 q}(X ; \mathbb{Z})$, we define

$$
\mathrm{GW}_{(m, J)}^{\beta}\left(\eta, \mu_{0} ; \mu\right)=\left\langle\eta \operatorname{ev}_{0}^{*}\left(\mu_{0} \mu_{J}\right) \prod_{j \notin J}\left(\psi_{j}^{c_{j}} \operatorname{ev}_{j}^{*} \mu_{j}\right),\left[\mathcal{Z}_{(m,[k]-J)}(X, \beta ; \mathcal{J})\right]^{\mathrm{vir}}\right\rangle \in \mathbb{Q} \text {. }
$$

Remark The exact definitions of standard and reduced GW-invariants are not essential for the purposes of the present paper, as our starting point in Section 3 will be Proposition 3.1 in [15], restated as Proposition 3.2 below, which gives a description of the difference between the two invariants. Roughly speaking, the standard GW-invariants are modeled on the moduli spaces $\overline{\mathfrak{M}}_{g, k}\left(\mathbb{P}^{n}, d\right)$ of stable maps to $\mathbb{P}^{n}$. The reduced genus-one invariants are modeled on the closure

$$
\overline{\mathfrak{M}}_{1, k}^{0}\left(\mathbb{P}^{n}, d\right) \subset \overline{\mathfrak{M}}_{1, k}\left(\mathbb{P}^{n}, d\right)
$$

of the locus consisting of genus-one maps from smooth domains. The standard invariants are counts of solutions $u$ of a perturbed $\bar{\partial}$-equation

$$
\bar{\partial} u+v(u)=0
$$


for a generic family of deformations $v$. The reduced genus-one invariants are also counts of solutions a perturbed $\bar{\partial}$-equation, but with a generic effectively supported family of deformations $v$; see Section 3.3. An algebro-geometric approach to reduced genus-one invariants is described at the end of [12], though it remains to be formally proved that the resulting invariants agree with [15]. The reduced invariants see genusone holomorphic curves, but not regular genus-zero holomorphic maps; the standard invariants do not separate the two. One of the key points of [15] is that the intersections of natural main strata of moduli spaces of genus-one stable maps do not contribute to the standard invariants, and thus these invariants can be split into contributions from the main strata.

\subsection{Main theorem}

The description of Proposition 3.1 in [15] implies that the difference between $\mathrm{GW}_{1, k}^{\beta}(\mu)$ and $\mathrm{GW}_{1, k}^{\beta ; 0}(\mu)$ is some linear combination of the invariants $\mathrm{GW}_{(m, J)}^{\beta}\left(\eta_{p}, c_{q}(T X) ; \mu\right)$ or equivalently of $\mathrm{GW}_{(m, J)}^{\beta}\left(\widetilde{\eta}_{p}, c_{q}(T X) ; \mu\right)$. The coefficients should be sums of products of top intersections of tautological classes on moduli spaces of genus-zero and genus-one curves, $\overline{\mathcal{M}}_{g, N}$. The simplest expressions in the first case, however, appear to be given by Hodge numbers on the blowups $\widetilde{\mathcal{M}}_{1,([m], J)}$ of $\overline{\mathcal{M}}_{1, m+|J|}$ constructed in Subsection 2.3 in [13] and involve the universal $\psi$-class

$$
\tilde{\psi} \equiv c_{1}(\widetilde{\mathbb{E}}) \in H^{2}\left(\widetilde{\mathcal{M}}_{1,([m], J)}\right)
$$

obtained by twisting the Hodge line bundle $\mathbb{E}$; see Section 3.1 below.

Thus, given finite sets $I$ and $J$, not both empty, and a tuple of integers $\left(\tilde{c},\left(c_{j}\right)_{j \in J}\right)$, we define

$$
\left\langle\widetilde{c} ;\left(c_{j}\right)_{j \in J}\right\rangle_{(I, J)}=\left\langle\tilde{\psi}^{\tilde{c}} \cdot \prod_{j \in J} \pi^{*} \psi_{j}^{c_{j}},\left[\widetilde{\mathcal{M}}_{1,(I, J)}\right]\right\rangle \in \mathbb{Q},
$$

where $\pi: \widetilde{\mathcal{M}}_{1,[[k], J)} \longrightarrow \overline{\mathcal{M}}_{1,[k] \sqcup J}$ is the blowdown map. If $\tilde{c}+\sum_{j \in J} c_{j} \neq|I|+|J|$, $\tilde{c}<0$, or $c_{j}<0$ for some $j \in J$, the number in (2-7) is zero. By Theorem 1.1 in [19], the numbers (2-7) satisfy:

(R1) If $i^{*} \in I$ and $c_{j}>0$ for all $j \in J$,

$$
\left\langle\widetilde{c} ;\left(c_{j}\right)_{j \in J}\right\rangle_{(I, J)}=\left\langle\widetilde{c} ;\left(c_{j}\right)_{j \in J}\right\rangle_{\left(I-i^{*}, J \sqcup i^{*}\right)} ;
$$


(R2) If $c_{j}=0$ for some $j^{*} \in J$,

$$
\begin{aligned}
\left\langle\tilde{c} ;\left(c_{j}\right)_{j \in J}\right\rangle_{(I, J)}=|I|\left\langle\tilde{c}-1 ;\left(c_{j}\right)_{j \in J-\left\{j^{*}\right\}}\right\rangle_{\left(I, J-j^{*}\right)} & \\
& +\sum_{j \in J-\left\{j^{*}\right\}}\left\langle\tilde{c} ; c_{j}-1,\left(c_{j^{\prime}}\right)_{j^{\prime} \in J-\left\{j^{*}, j\right\}}\right\rangle_{\left(I, J-j^{*}\right)} .
\end{aligned}
$$

Along with the relation

$$
\langle 0 ; \underbrace{1,1, \ldots, 1}_{m}\rangle_{(0,[m])} \equiv\left\langle\psi_{1} \ldots \psi_{m},\left[\overline{\mathcal{M}}_{1, m}\right]\right\rangle=\frac{(m-1) !}{24},
$$

which follows from the usual dilation relation [5, Section 26.2], the two recursions completely determine the numbers (2-7). In particular (see Corollary 1.2 in [19]):

$$
\langle|I|+|J| ; 0\rangle_{(I, J)} \equiv\left\langle\tilde{\psi}^{|I|+|J|},\left[\widetilde{\mathcal{M}}_{1,(I, J)}\right]\right\rangle=\frac{1}{24} \cdot|I|^{|J|} \cdot(|I|-1) !
$$

Theorem 1A If $(X, \omega)$ is a compact symplectic manifold of real dimension $2 n$, $k \in \overline{\mathbb{Z}}^{+}, \beta \in H_{2}(X ; \mathbb{Z})-0$, and $\mu$ is as in (2-3) and (2-4), then

$$
\begin{aligned}
\mathrm{GW}_{1, k}^{\beta}(\mu)-\mathrm{GW}_{1, k}^{\beta ; 0}(\mu) & =\sum_{m \in \mathbb{Z}^{+}} \sum_{J \subset[k]}\left((-1)^{m+|J|-p_{J}(\mu)}\left(m+|J|-p_{J}(\mu) ;\left(c_{j}\right)_{j \in J}\right)_{([m], J)}\right. \\
& \left.\times \sum_{p=0}^{d_{m, J}(\mu)} \mathrm{GW}_{(m, J)}^{\beta}\left(\eta_{p}, c_{d_{m, J}(\mu)-p}(T X) ; \mu\right)\right) .
\end{aligned}
$$

The sum in $(2-10)$ is finite because $\mathcal{Z}_{(m,[k]-J)}(X, \beta ; \mathcal{J})$ is empty if $\langle\omega, \beta\rangle / m$ is smaller than the minimal energy of a nonconstant $\mathcal{J}$-holomorphic map $S^{2} \longrightarrow X$. Therefore, $\mathrm{GW}_{(m, J)}^{\beta}$ is zero if $\langle\omega, \beta\rangle / m$ is smaller than the minimal energy of a nonconstant $\mathcal{J}$-holomorphic map $S^{2} \longrightarrow X$ for any $\omega$-compatible almost complex structure $\mathcal{J}$. Theorem 1 A is proved in Section 3.

We next express the difference between $\mathrm{GW}_{1, k}^{\beta}(\mu)$ and $\mathrm{GW}_{1, k}^{\beta ; 0}(\mu)$ in terms of the numbers $\mathrm{GW}_{(m, J)}^{\beta}\left(\widetilde{\eta}_{p}, c_{q}(T X) ; \mu\right)$. If $J$ is a finite set, $\mathbf{c} \equiv\left(c_{j}\right)_{j \in J}$ is a $J$-tuple of integers, and $J_{0} \subset J$, let

$$
p_{J_{0}}(\mathbf{c})=\sum_{j \in J_{0}} c_{j} \in \mathbb{Z}
$$


If in addition $m \in \mathbb{Z}^{+}$, we define

$$
\begin{aligned}
\Theta_{m, J}(\mathbf{c})=\sum_{J=\bigsqcup_{i=0}^{i=m} J_{i}}\left((-1)^{m+\left|J_{0}\right|-p_{J_{0}}(\mathbf{c})}\left(m+|J|-p_{J_{0}}(\mathbf{c}) ;\left(c_{j}\right)_{j \in J_{0}}\right)_{\left([m], J_{0}\right)}\right. & \\
& \left.\times \prod_{i=1}^{i=m}\left(\begin{array}{c}
\left|J_{i}\right|-1 \\
\left(c_{j}\right)_{j \in J_{i}}
\end{array}\right)\right),
\end{aligned}
$$

where $\quad\left(\begin{array}{c}\left|J_{i}\right|-1 \\ \left(c_{j}\right)_{j \in J_{i}}\end{array}\right) \equiv\left(\begin{array}{c}\left|J_{i}\right|-1 \\ \left(c_{j}\right)_{j \in J_{i}},\left|J_{i}\right|-1-p_{J_{i}}(\mathbf{c})\end{array}\right), \quad\left(\begin{array}{c}-1 \\ (\cdot)\end{array}\right) \equiv 1$.

The multinomial coefficients above appear as Hodge numbers on $\overline{\mathcal{M}}_{0,\left|J_{i}\right|+2}$.

Along with the relation

$$
\left(\begin{array}{c}
N \\
c_{1}, c_{2}, \ldots, c_{l}
\end{array}\right)=\left(\begin{array}{c}
N-1 \\
c_{1}-1, c_{2}, \ldots, c_{l}
\end{array}\right)+\ldots+\left(\begin{array}{c}
N-1 \\
c_{1}, c_{2}, \ldots, c_{l-1}, c_{l}-1
\end{array}\right),
$$

the recursions $(R 1)$ and $(R 2)$ imply that the numbers in (2-11) satisfy

( $\widetilde{R} 1)$ If $m>1$ and $c_{j}>1$ for all $j \in J$,

$$
\Theta_{m, J}\left(\left(c_{j}\right)_{j \in J}\right)=-(m-1) \Theta_{m-1, J}\left(\left(c_{j}\right)_{j \in J}\right)+\sum_{j \in J} \Theta_{m-1, J}\left(c_{j}-1,\left(c_{j^{\prime}}\right)_{j^{\prime} \in J-j}\right) ;
$$

( $\widetilde{R} 2)$ If $c_{j *}=0$ for some $j^{*} \in J$,

$$
\Theta_{m, J}\left(\left(c_{j}\right)_{j \in J}\right)=\sum_{j \in J-\left\{j^{*}\right\}} \Theta_{m, J-j^{*}}\left(c_{j}-1,\left(c_{j^{\prime}}\right)_{j^{\prime} \in J-\left\{j^{*}, j\right\}}\right) .
$$

Along with the relation

$$
\Theta_{1, \varnothing}()=-\frac{1}{24},
$$

which follows from $(2-8),(\widetilde{R} 1)$ and $(\widetilde{R} 2)$ are sufficient to determine $\Theta_{m, J}\left(\left(c_{j}\right)_{j \in J}\right)$ in many cases. In particular,

$$
\Theta_{m, J}\left(\left(c_{j}\right)_{j \in J}\right)=\frac{(-1)^{m}(m-1) !}{24} \begin{cases}1, & \text { if } J=\varnothing ; \\ 0, & \text { if } \sum_{j \in J} c_{j}<|J| .\end{cases}
$$

The original recursions $(R 1)$ and $(R 2)$ are sufficient to compute $\Theta_{m, J}\left(\left(c_{j}\right)_{j \in J}\right)$ in all cases. However, it is more convenient to make use of the remaining relation of Theorem 1.1 in [19]: if $c_{j}=1$ for some $j^{*} \in J$, then

$$
\left\langle\widetilde{c} ;\left(c_{j}\right)_{j \in J}\right\rangle_{(I, J)}=(|I|+|J|-1)\left\langle\widetilde{c} ;\left(c_{j}\right)_{j \in J-j^{*}}\right\rangle_{\left(I, J-j^{*}\right)} .
$$

This gives us a third relation for the numbers $(2-11)$ : 
( $\widetilde{R} 3)$ If $c_{j}=1$ for some $j^{*} \in J$,

$$
\Theta_{m, J}\left(\left(c_{j}\right)_{j \in J}\right)=(m+|J|-1) \Theta_{m, J-j^{*}}\left(\left(c_{j}\right)_{j \in J-j^{*}}\right) .
$$

The three relations $(\widetilde{R} 1)-(\widetilde{R} 3)$, along with the initial condition $\Theta_{1, \varnothing}()=-1 / 24$, determine the numbers $\Theta_{m, J}(\mathbf{c})$ completely.

Theorem 1B If $(X, \omega)$ is a compact symplectic manifold of real dimension $2 n$, $k \in \overline{\mathbb{Z}}^{+}, \beta \in H_{2}(X ; \mathbb{Z})-0$, and $\mu$ is as in (2-3) and (2-4), then

$$
\begin{aligned}
\mathrm{GW}_{1, k}^{\beta}(\mu)-\mathrm{GW}_{1, k}^{\beta ; 0}(\mu)= & \sum_{m \in \mathbb{Z}^{+}} \sum_{J \subset[k]}\left(\Theta_{m, J}\left(\left(c_{j}\right)_{j \in J}\right)\right. \\
& \left.\times \sum_{p=0}^{d_{m, J}(\mu)} \mathrm{GW}_{(m, J)}^{\beta}\left(\tilde{\eta}_{p}, c_{d_{m, J}(\mu)-p}(T X) ; \mu\right)\right) .
\end{aligned}
$$

This theorem follows immediately from Theorem 1A above and Lemma 2.1 below. In turn, the latter follows from Lemma 2.2.1 and Subsection 3.2 in [11]; see also Subsection 3.3 in [18]. ${ }^{2}$

Lemma 2.1 Suppose $(X, \omega)$ is a compact symplectic manifold of real dimension $2 n$, $k \in \overline{\mathbb{Z}}^{+}, \beta \in H_{2}(X ; \mathbb{Z})-0$, and $\mu$ is as in (2-3) and (2-4). If $m \in \mathbb{Z}^{+}, p \in \overline{\mathbb{Z}}^{+}$, and $J \subset[k]$,

$$
\begin{aligned}
\mathrm{GW}_{(m, J)}^{\beta}\left(\eta_{p}, c_{d_{m, J}}(\mu)-p\right. & (T X) ; \mu) \\
= & \sum_{J \subset J^{\prime} \subset[k]}\left(\sum_{J^{\prime}-J=\bigsqcup_{i=1}^{i=m} J_{i}} \prod_{i=1}^{i=m}\left(\begin{array}{c}
\left|J_{i}\right|-1 \\
\left(c_{j}\right)_{j \in J_{i}}
\end{array}\right)\right. \\
& \left.\quad \times \mathrm{GW}_{\left(m, J^{\prime}\right)}^{\beta}\left(\tilde{\eta}_{p-\left|J^{\prime}-J\right|+p_{J^{\prime}-J}(\mu), c_{d_{m, J}}(\mu)-p}(T X) ; \mu\right)\right) .
\end{aligned}
$$

The advantage of Theorem 1B over Theorem 1A is that the coefficients of the genuszero GW-invariants in (2-14) satisfy simpler recursions and are more likely to vanish,

\footnotetext{
${ }^{2}$ Lemma 2.1 is a consequence of the following identities. If $J_{0} \subset J$ is nonempty, let $D_{J_{0}} \subset$ $\overline{\mathfrak{M}}_{0,0 \sqcup J}(X, \beta ; \mathcal{J})$ be the (virtual) divisor whose (virtually) generic element is a map from a union of two $\mathbb{P}^{1}$ 's, one of which is contracted and carries the marked points indexed by the set $0 \sqcup J_{0}$. In particular, $D_{J_{0}} \approx \overline{\mathcal{M}}_{0,\{0,1\} \sqcup J_{0}} \times \overline{\mathfrak{M}}_{0,0 \sqcup\left(J-J_{0}\right)}(X, \beta ; \mathcal{J})$. If $\pi_{P}$ and $\pi_{B}$ are the two component projection maps, then $\psi_{0}=\widetilde{\psi}_{0}+\sum_{\varnothing \neq J_{0} \subset J} D_{J_{0}},\left.\psi_{0}\right|_{D_{J_{0}}}=\pi_{P}^{*} \psi_{0},\left.\tilde{\psi}_{0}\right|_{D_{J_{0}}}=\pi_{B}^{*} \tilde{\psi}_{0}$.
} 
due to (2-12). For example, if $c_{j}=0$ for all $j$, ie there are no descendant classes involved, (2-14) reduces to

$$
\begin{aligned}
& \mathrm{GW}_{1, k}^{\beta}(\mu)-\mathrm{GW}_{1, k}^{\beta ; 0}(\mu) \\
& =\frac{1}{24} \sum_{m=1}^{2 m \leq n}\left((-1)^{m}(m-1) ! \sum_{p=0}^{n-2 m} \mathrm{GW}_{(m, \varnothing)}^{\beta}\left(\tilde{\eta}_{p}, c_{n-2 m-p}(T X) ; \mu\right)\right) .
\end{aligned}
$$

This formula looks remarkably similar to the formula for the correction term in Theorem 1.1 in [18] enumerating one-nodal rational curves. ${ }^{3}$ This is not too surprising as both expressions arise from counting zeros of analogous affine bundle maps; see Section 3.4.

\subsection{Genus-one GW-invariants of Calabi-Yau hypersurfaces}

The essence of mirror symmetric predictions for GW-invariants of Calabi-Yau manifolds is that these invariants can be expressed in terms of certain hypergeometric series. In this subsection, we deduce a mirror symmetry type of formula for the standard genus-one GW-invariants of Calabi-Yau projective hypersurfaces from a formula for the reduced genus-one GW-invariants obtained in [16], (2-15), and a formula for genus-zero GWinvariants confirmed in [5, Chapter 30]. In particular, we show that the difference between the two invariants, ie (2-15), simply cancels the last term in Corollary 3.5 in [16]. The $n \leq 5$ cases of Theorem 2 below have already been obtained [16], with the $n=5$ case confirming the prediction of [1]. The $n=6$ case confirms the prediction of [6].

Fix an integer $n \geq 3$ and let $X \subset \mathbb{P}^{n-1}$ be a smooth degree- $n$ hypersurface. In this case, $d_{1,0}(X, \beta)=0$ for every $\beta \in H_{2}(X ; \mathbb{Z})$. For each $d \in \mathbb{Z}^{+}$, denote by $N_{1}^{d}(X) \in \mathbb{Q}$ and $N_{1}^{d ; 0}(X) \in \mathbb{Q}$ the standard and reduced degree- $d$ genus-one GW-invariant of $X \subset \mathbb{P}^{n-1}$, ie the sum of $\mathrm{GW}_{1,0}^{\beta}(\varnothing)$ and $\mathrm{GW}_{1,0}^{\beta ; 0}(\varnothing)$, respectively, over all $\beta$ lying in the preimage of $d \ell$ under the natural homomorphism

$$
H_{2}(X ; \mathbb{Z}) \longrightarrow H_{2}\left(\mathbb{P}^{n-1} ; \mathbb{Z}\right),
$$

where $\ell \in H_{2}\left(\mathbb{P}^{n-1} ; \mathbb{Z}\right)$ is the homology class of the line.

For each $q=0,1, \ldots$, define $I_{0, q}(t)$ by

$$
\sum_{q=0}^{\infty} I_{0, q}(t) w^{q} \equiv e^{w t} \sum_{d=0}^{\infty} e^{d t} \frac{\prod_{r=1}^{r=n d}(n w+r)}{\prod_{r=1}^{r=d}(w+r)^{n}} \equiv R(w, t) .
$$

\footnotetext{
${ }^{3}$ In [18], the meanings of $\eta_{p}$ and $\tilde{\eta}_{p}$ are reversed.
} 
Each $I_{0, q}(t)$ is a degree- $q$ polynomial in $t$ with coefficients that are power series in $e^{t}$. For example,

$$
I_{0}(t)=1+\sum_{d=1}^{\infty} e^{d t} \frac{(n d) !}{(d !)^{n}}, \quad I_{1}(t)=t I_{0}(t)+\sum_{d=1}^{\infty} e^{d t}\left(\frac{(n d) !}{(d !)^{n}} \sum_{r=d+1}^{n d} \frac{n}{r}\right)
$$

For $p, q \in \mathbb{Z}^{+}$with $q \geq p$, let

$$
I_{p, q}(t)=\frac{d}{d t}\left(\frac{I_{p-1, q}(t)}{I_{p-1, p-1}(t)}\right)
$$

It is straightforward to check that each of the "diagonal" terms $I_{p, p}(t)$ is a power series in $e^{t}$ with constant term 1 , whenever it is defined. In particular, the division in (2-18) is well-defined for all $p$. A definition of $I_{p, p}(t)$ involving power series in $q=e^{t}$ only (and not in $t$ ) and a number of relations between the power series $I_{p, p}(t)$ can be found in [14]. Let

$$
T=\frac{I_{0,1}(t)}{I_{0,0}(t)}
$$

By (2-17), the map $t \longrightarrow T$ is a change of variables; it will be called the mirror map.

Theorem 2 The genus-one degree- $d$ Gromov-Witten invariants of a degree- $n$ hypersurface $X$ in $\mathbb{P}^{n-1}$ are given by

$$
\begin{aligned}
& \sum_{d=1}^{\infty} e^{d T} N_{1}^{d}(X) \\
& =\left(\frac{(n-2)(n+1)}{48}+\frac{1-(1-n)^{n}}{24 n^{2}}\right)(T-t)+\frac{n^{2}-1+(1-n)^{n}}{24 n} \ln I_{0,0}(t) \\
& - \begin{cases}\frac{n-1}{48} \ln \left(1-n^{n} e^{t}\right)+\sum_{p=0}^{(n-3) / 2} \frac{(n-1-2 p)^{2}}{8} \ln I_{p, p}(t), & \text { if } 2 \not n ; \\
\frac{n-4}{48} \ln \left(1-n^{n} e^{t}\right)+\sum_{p=0}^{(n-4) / 2} \frac{(n-2 p)(n-2-2 p)}{8} \ln I_{p, p}(t), & \text { if } 2 \mid n,\end{cases}
\end{aligned}
$$

where $t$ and $T$ are related by the mirror map (2-19).

The distinction between the $n$ odd and $n$ even cases appears because the formula of Corollary 3.5 in [16] uses the reflection symmetry property of Theorem 2 in [14] to reduce the number of different power series $I_{p, p}$ used. A uniform formula can be obtained from Theorem 3.3 in [16]. 
Let $\bar{R}(w, t)=R(w, t) / I_{0,0}(t)$. Then, $e^{-w t} \bar{R}(w, t)$ is a power series with $e^{t}$-constant term 1 and

$$
\left.\mathcal{D}_{w}^{p} \ln \bar{R}(w, t) \equiv \frac{1}{p !}\left\{\frac{d}{d w}\right\}^{p}(\ln (\bar{R}(w, t)))\right|_{w=0} \in \mathbb{Q}\left[\left[e^{t}\right]\right]
$$

for all $p \in \mathbb{Z}^{+}$with $p \geq 2$. Theorem 2 follows immediately from Corollary 3.4 in [16], which we restate below, (2-14), and Lemma 2.2. Note that since $\operatorname{dim} X=n-2$ and $k=0,(2-15)$ can be written as

$$
N_{1}^{d}(X)-N_{1}^{d ; 0}(X)=\frac{1}{24} \sum_{p=2}^{n-2} \sum_{m=1}^{2 m \leq p}(-1)^{m}(m-1) ! \mathrm{GW}_{(m, \varnothing)}^{d}\left(\eta_{p-2 m}, c_{n-2-p}(T X) ; \varnothing\right) .
$$

Theorem [16, Corollary 3.4] The reduced genus-one degree-d Gromov-Witten invariants of a degree- $n$ hypersurface $X$ in $\mathbb{P}^{n-1}$ are given by

$$
\begin{aligned}
& \sum_{d=1}^{\infty} e^{d T} N_{1}^{d ; 0}(X) \\
& =\left(\frac{(n-2)(n+1)}{48}+\frac{1-(1-n)^{n}}{24 n^{2}}\right)(T-t)+\frac{n^{2}-1+(1-n)^{n}}{24 n} \ln I_{0,0}(t) \\
& - \begin{cases}\frac{n-1}{48} \ln \left(1-n^{n} e^{t}\right)+\sum_{p=0}^{(n-3) / 2} \frac{(n-1-2 p)^{2}}{8} \ln I_{p, p}(t), & \text { if } 2 \not n \\
\frac{n-4}{48} \ln \left(1-n^{n} e^{t}\right)+\sum_{p=0}^{(n-4) / 2} \frac{\frac{(n-2 p)(n-2-2 p)}{8} \ln I_{p, p}(t),}{} & \text { if } 2 \mid n ;\end{cases} \\
& +\frac{n}{24} \sum_{p=2}^{n-2}\left(\mathcal{D}_{w}^{n-2-p} \frac{(1+w)^{n}}{(1+n w)}\right)\left(\mathcal{D}_{w}^{p} \ln \bar{R}(w, t)\right),
\end{aligned}
$$

where $t$ and $T$ are related by the mirror map (2-19).

Lemma 2.2 If $X \subset \mathbb{P}^{n-1}$ is a degree- $n$ hypersurface, $x \in H^{2}\left(\mathbb{P}^{n-1} ; \mathbb{Z}\right)$ is the hyperplane class, and $p, q \in \overline{\mathbb{Z}}^{+}$with $2 \leq p \leq n-2$,

$$
\begin{aligned}
& c_{q}(T X)=\left.\left(\mathcal{D}_{w}^{q} \frac{(1+w)^{n}}{(1+n w)}\right) x^{q}\right|_{X}, \\
& \sum_{d=1}^{\infty} e^{d T}\left(\sum_{m=1}^{2 m \leq p}(-1)^{m}(m-1) ! \mathrm{GW}_{(m, \varnothing)}^{d}\left(\eta_{p-2 m}, x^{n-2-p} ; \varnothing\right)\right) \\
& =-n \mathcal{D}_{w}^{p} \ln \bar{R}(w, t),
\end{aligned}
$$

if $T$ and $t$ are related by the mirror map (2-19). 
The relation (2-20) is immediate from $c\left(T \mathbb{P}^{n-1}\right)=(1+x)^{n}$. We deduce (2-21) below from the conclusion of Chapter 30 in [5].

Let $\mathfrak{U}$ be the universal curve over $\overline{\mathfrak{M}}_{(m, \varnothing)}\left(\mathbb{P}^{n-1}, d\right)$, with structure map $\pi$ and evaluation map ev:

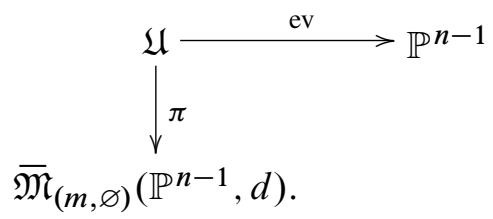

In other words, the fiber of $\pi$ over a tuple $\left(\left[\mathcal{C}_{i}, u_{i}\right]\right)_{i \in[m]}$ is the wedge of curves $\mathcal{C}_{i}$ identified at the marked point $x_{0}$ of each of the curves, while

$$
\mathrm{ev}\left(\left[\left(\mathcal{C}_{i}, u_{i}\right)_{i \in[m]}, z\right]\right)=u_{i}(z) \quad \text { if } \quad z \in \mathcal{C}_{i}
$$

The orbi-sheaf

$$
\pi_{*} \operatorname{ev}^{*} \mathcal{O}_{\mathbb{P}^{n-1}}(n) \longrightarrow \overline{\mathfrak{M}}_{(m, \varnothing)}\left(\mathbb{P}^{n-1}, d\right)
$$

is locally free; it is the sheaf of (holomorphic) sections of the vector orbi-bundle

$$
\mathcal{V}_{(m, \varnothing)} \equiv \overline{\mathfrak{M}}_{(m, \varnothing)}(\mathcal{L}, d) \longrightarrow \overline{\mathfrak{M}}_{(m, \varnothing)}\left(\mathbb{P}^{n-1}, d\right),
$$

where $\mathcal{L} \longrightarrow \mathbb{P}^{n-1}$ is the total space of the line bundle corresponding to the sheaf $\mathcal{O}_{\mathbb{P}^{n-1}}(n)$. By the (genus-zero) hyperplane-section relation,

$$
\begin{aligned}
(m-1) ! \mathrm{GW}_{(m, \varnothing)}^{d} & \left(\eta_{p-2 m}, x^{n-2-p} ; \varnothing\right) \\
= & \frac{1}{m}\left\langle\eta_{p-2 m} \mathrm{ev}_{0}^{*} x^{n-2-p} e\left(\mathcal{V}_{(m, \varnothing)}\right),\left[\overline{\mathfrak{M}}_{(m, \varnothing)}\left(\mathbb{P}^{n-1}, d\right)\right]\right\rangle .
\end{aligned}
$$

The $m=1$ case of (2-22) is completely standard, and the same argument applies in all cases.

There is a natural surjective bundle homomorphism

$$
\widetilde{\mathrm{ev}}_{0}: \mathcal{V}_{(1, \varnothing)} \longrightarrow \mathrm{ev}_{0}^{*} \mathcal{O}_{\mathbb{P}^{n-1}}(n), \quad([\mathcal{C}, u, \xi]) \longrightarrow \xi\left(x_{0}(\mathcal{C})\right)
$$

over $\overline{\mathfrak{M}}_{(1, \varnothing)}\left(\mathbb{P}^{n-1}, d\right) \equiv \overline{\mathfrak{M}}_{0,\{0\}}\left(\mathbb{P}^{n-1}, d\right)$. Thus,

$$
\mathcal{V}_{(1, \varnothing)}^{\prime} \equiv \operatorname{ker} \widetilde{\mathrm{ev}}_{0} \longrightarrow \overline{\mathfrak{M}}_{(1, \varnothing)}\left(\mathbb{P}^{n-1}, d\right)
$$

is a vector orbi-bundle. It is straightforward to see that

$$
e\left(\mathcal{V}_{(m, \varnothing)}\right)=n \operatorname{ev}_{0}^{*} x \prod_{i=1}^{i=m} \pi_{i}^{*} e\left(\mathcal{V}_{(1, \varnothing)}^{\prime}\right)
$$


For each $r \in \overline{\mathbb{Z}}^{+}$, let

$$
Z_{r}\left(e^{T}\right)=\sum_{d=1}^{\infty} e^{d T}\left\langle\psi_{0}^{r} \mathrm{ev}_{0}^{*} x^{n-3-r} e\left(\mathcal{V}_{(1, \varnothing)}^{\prime}\right),\left[\overline{\mathfrak{M}}_{(1, \varnothing)}\left(\mathbb{P}^{n-1}, d\right)\right]\right\rangle
$$

Using the string relation [5, Section 26.3], the conclusion of [5, Chapter 30] can be reformulated as

$$
e^{T w}\left(1+\sum_{r=0}^{n-3} Z_{r}\left(e^{T}\right) w^{r+2}\right)=\bar{R}(w, t) \in \mathbb{Q}[w]\left[\left[e^{t}\right]\right] / w^{n},
$$

with $T$ and $t$ related by the mirror map (2-19) as before.

We now verify (2-21). By (2-22), (2-23), and the decomposition along the small diagonal in $\left(\mathbb{P}^{n-1}\right)^{m}$, the left-hand side of (2-21) equals

$$
\begin{aligned}
& n \sum_{d=1}^{\infty} e^{d T}\left(\sum_{m=1}^{2 m \leq p} \frac{(-1)^{m}}{m}\left\langle\mathrm{ev}_{0}^{*} x^{n-1-p} \prod_{i=1}^{i=m} \pi_{i}^{*} \frac{e\left(\mathcal{V}_{(1, \varnothing)}^{\prime}\right)}{1-\psi_{0}},\left[\overline{\mathfrak{M}}_{(m, \varnothing)}\left(\mathbb{P}^{n-1}, d\right)\right]\right\rangle\right) \\
& =n \sum_{m=1}^{2 m \leq p} \frac{(-1)^{m}}{m} \sum_{d=1}^{\infty} e^{d T} \\
& \times\left(\sum_{\substack{i=m \\
\sum_{i=1}^{i=1} d_{i}=d \\
d_{i}>0}} \sum_{\substack{i=m \\
\sum_{i=1}^{i=1} p_{i}=p \\
p_{i} \geq 0}} \prod_{i=1}^{i=m}\left\langle\mathrm{ev}_{0}^{*} x^{n-1-p_{i}} \frac{e\left(\mathcal{V}_{(1, \varnothing)}^{\prime}\right)}{1-\psi_{0}},\left[\overline{\mathfrak{M}}_{(1, \varnothing)}\left(\mathbb{P}^{n-1}, d_{i}\right)\right]\right\rangle\right) \\
& =n \sum_{m=1}^{2 m \leq p} \frac{(-1)^{m}}{m} \sum_{\substack{i=m \\
\sum_{i=1}^{i=1} p_{i}=p \\
p_{i} \geq 2}} \prod_{i=1}^{i=m} Z_{p_{i}-2}\left(e^{T}\right)=-n \mathcal{D}_{w}^{p} \ln \left(1+\sum_{r=0}^{n-3} Z_{r}\left(e^{T}\right) w^{r+2}\right) .
\end{aligned}
$$

The relation (2-21) now follows from (2-24).

\section{Proof of Theorem 1A}

\subsection{Blowups of moduli spaces of genus-one curves}

In this subsection we review some aspects of the blowup construction of Subsection 2.3 in [13] and add new ones, which will be used in Section 3.4.

If $I$ is a finite set, let

$$
\mathcal{A}_{1}(I)=\left\{\left(I_{P},\left\{I_{k}: k \in K\right\}\right): K \neq \varnothing ; I=\bigsqcup_{k \in\{P\} \sqcup K} I_{k} ;\left|I_{k}\right| \geq 2 \forall k \in K\right\} .
$$


Here $P$ stands for "principal" (component). If $\rho=\left(I_{P},\left\{I_{k}: k \in K\right\}\right)$ is an element of $\mathcal{A}_{1}(I)$, we denote by $\mathcal{M}_{1, \rho}$ the subset of $\overline{\mathcal{M}}_{1, I}$ consisting of the stable curves $\mathcal{C}$ such that

(i) $\mathcal{C}$ is a union of a smooth torus and $|K|$ projective lines, indexed by $K$;

(ii) each line is attached directly to the torus;

(iii) for each $k \in K$, the marked points on the line corresponding to $k$ are indexed by $I_{k}$.

For example, the first diagram in Figure 2 shows an element of $\mathcal{M}_{1, \rho}$ with

$$
\rho=\left(\left\{i_{1}, i_{2}\right\},\left\{\left\{i_{3}, i_{4}, i_{5}, i_{6}\right\},\left\{i_{7}, i_{8}, i_{9}\right\}\right\}\right) ;
$$

the number next to each component indicates the genus. Let $\overline{\mathcal{M}}_{1, \rho}$ be the closure of $\mathcal{M}_{1, \rho}$ in $\overline{\mathcal{M}}_{1, I}$. It is well-known that each space $\overline{\mathcal{M}}_{1, \rho}$ is a smooth subvariety of $\overline{\mathcal{M}}_{1, I}$.
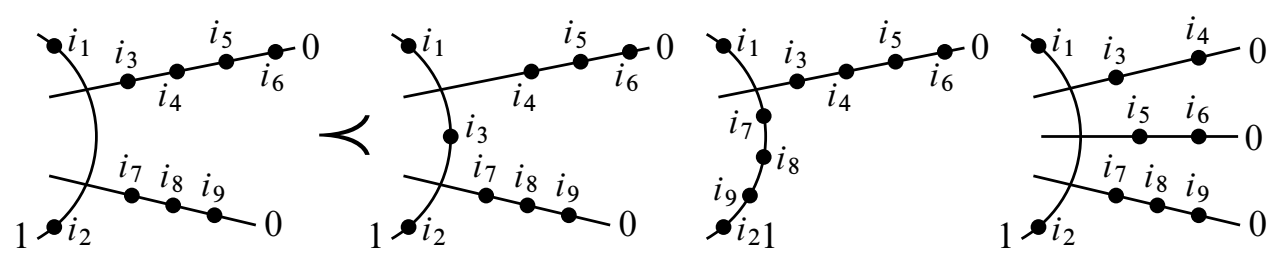

$I_{P}=\left\{i_{1}, i_{2}\right\}, K=\{1,2\}$

$I_{1}=\left\{i_{3}, i_{4}, i_{5}, i_{6}\right\}, I_{2}=\left\{i_{7}, i_{8}, i_{9}\right\}$

Figure 2: Examples of partial ordering (3-2)

We define a partial ordering on the set $\mathcal{A}_{1}(I) \sqcup\{(I, \varnothing)\}$ by setting

$$
\rho^{\prime} \equiv\left(I_{P}^{\prime},\left\{I_{k}^{\prime}: k \in K^{\prime}\right\}\right) \prec \rho \equiv\left(I_{P},\left\{I_{k}: k \in K\right\}\right)
$$

if $\rho^{\prime} \neq \rho$ and there exists a map $\varphi: K \longrightarrow K^{\prime}$ such that $I_{k} \subset I_{\varphi(k)}^{\prime}$ for all $k \in K$. This condition means that the elements of $\mathcal{M}_{1, \rho^{\prime}}$ can be obtained from the elements of $\mathcal{M}_{1, \rho}$ by moving more points onto the bubble components or combining the bubble components; see Figure 2.

Let $I$ and $J$ be finite sets such that $I$ is not empty and $|I|+|J| \geq 2$. We put

$$
\mathcal{A}_{1}(I, J)=\left\{\left(\left(I_{P} \sqcup J_{P}\right),\left\{I_{k} \sqcup J_{k}: k \in K\right\}\right) \in \mathcal{A}_{1}(I \sqcup J): I_{k} \neq \varnothing \forall k \in K\right\} .
$$


Note that if $\rho \in \mathcal{A}_{1}(I \sqcup J)$, then $\rho \in \mathcal{A}_{1}(I, J)$ if and only if every bubble component of an element of $\mathcal{M}_{1, \rho}$ carries at least one element of $I$. The partially ordered set $\left(\mathcal{A}_{1}(I, J), \prec\right)$ has a unique minimal element

$$
\rho_{\min } \equiv(\varnothing,\{I \sqcup J\}) .
$$

Let $<$ be an ordering on $\mathcal{A}_{1}(I, J)$ extending the partial ordering $\prec$. We denote the corresponding maximal element by $\rho_{\max }$. If $\rho \in \mathcal{A}_{1}(I, J)$, define

$$
\rho-1= \begin{cases}\max \left\{\rho^{\prime} \in \mathcal{A}_{1}(I, J): \rho^{\prime}<\rho\right\}, & \text { if } \rho \neq \rho_{\min } \\ 0, & \text { if } \rho=\rho_{\text {min }},\end{cases}
$$

where the maximum is taken with respect to the ordering $<$.

The starting data for the blowup construction of Subsection 2.3 in [13] is given by

$$
\overline{\mathcal{M}}_{1,(I, J)}^{0}=\overline{\mathcal{M}}_{1, I \sqcup J}, \quad \mathbb{E}_{0}=\mathbb{E} \longrightarrow \overline{\mathcal{M}}_{1,(I, J)}^{0} .
$$

Suppose $\rho \in \mathcal{A}_{1}(I, J)$ and we have constructed

(I1) a blowup $\pi_{\rho-1}: \overline{\mathcal{M}}_{1,(I, J)}^{\rho-1} \longrightarrow \overline{\mathcal{M}}_{1,(I, J)}^{0}$ of $\overline{\mathcal{M}}_{1,(I, J)}^{0}$ such that $\pi_{\rho-1}$ is one-toone outside of the preimages of the spaces $\overline{\mathcal{M}}_{1, \rho^{\prime}}^{0}$ with $\rho^{\prime} \leq \rho-1$;

(I2) a line bundle $\mathbb{E}_{\rho-1} \longrightarrow \overline{\mathcal{M}}_{1,(I, J)}^{\rho-1}$.

For each $\rho^{*}>\rho-1$, let $\overline{\mathcal{M}}_{1, \rho^{*}}^{\rho-1}$ be the proper transform of $\overline{\mathcal{M}}_{1, \rho^{*}}$ in $\overline{\mathcal{M}}_{1,(I, J)}^{\rho-1}$.

If $\rho \in \mathcal{A}_{1}(I, J)$ is as above, let

$$
\tilde{\pi}_{\rho}: \overline{\mathcal{M}}_{1,(I, J)}^{\rho} \longrightarrow \overline{\mathcal{M}}_{1,(I, J)}^{\rho-1}
$$

be the blowup of $\overline{\mathcal{M}}_{1,(I, J)}^{\rho-1}$ along $\overline{\mathcal{M}}_{1, \rho}^{\rho-1}$. We denote by $\overline{\mathcal{M}}_{1, \rho}^{\rho}$ the corresponding exceptional divisor and define

$$
\mathbb{E}_{\rho}=\tilde{\pi}_{\rho}^{*} \mathbb{E}_{\rho-1} \otimes \mathcal{O}\left(\overline{\mathcal{M}}_{1, \rho}^{\rho}\right) .
$$

It is immediate that the requirements (I1) and (I2), with $\rho-1$ replaced by $\rho$, are satisfied.

The blowup construction is concluded after $\left|\rho_{\max }\right|$ steps. Let

$$
\widetilde{\mathcal{M}}_{1,(I, J)}=\overline{\mathcal{M}}_{1,(I, J)}^{\rho_{\max }}, \quad \widetilde{\mathbb{E}}=\mathbb{E}_{\rho_{\max }}, \quad \tilde{\psi}=c_{1}(\widetilde{\mathbb{E}}) .
$$

By Lemma 2.6 in [13], the end result of this blowup construction is well-defined, ie independent of the choice of an ordering $<$ extending the partial ordering $\prec$. The 
reason is that different extensions of the partial order $\prec$ correspond to different orders of blowups along disjoint subvarieties. ${ }^{4}$

Remark If $I=\varnothing$ or $|I|+|J|=1$, we define $\widetilde{\mathcal{M}}_{1,(I, J)}=\overline{\mathcal{M}}_{1, I \sqcup J}$ and $\widetilde{\mathbb{E}}=\mathbb{E}$.

We next define natural line bundle homomorphisms $s_{i}: L_{i} \longrightarrow \mathbb{E}^{*}$ over $\overline{\mathcal{M}}_{1, I}$, where $L_{i} \longrightarrow \overline{\mathcal{M}}_{1, I}$ is the universal tangent line bundle at the $i$-th marked point. These homomorphisms will then be twisted to isomorphisms $\widetilde{s}_{i}$ on $\widetilde{\mathcal{M}}_{1,(I, J)}$. The homomorphism $s_{i}$ is induced by the natural pairing of tangent vectors and cotangent vectors at the $i$-th marked point. Explicitly,

$$
\left\{s_{i}([\mathcal{C} ; v])\right\}([\mathcal{C}, \psi])=\psi_{x_{i}(\mathcal{C})} v
$$

if $\quad[\mathcal{C}] \in \overline{\mathcal{M}}_{1, I},\left.\quad[\mathcal{C}, v] \in L_{i}\right|_{\mathcal{C}}=T_{x_{i}(\mathcal{C})} \mathcal{C},\left.\quad[\mathcal{C}, \psi] \in \mathbb{E}\right|_{\mathcal{C}}=H^{0}\left(\mathcal{C} ; T^{*} \mathcal{C}\right)$,

and $x_{i}(\mathcal{C}) \in \mathcal{C}$ is the marked point on $\mathcal{C}$ labeled by $i$. The homomorphism $s_{i}$ vanishes precisely on the curves for which the $i$-th marked point lies on a bubble component. In fact, as divisors,

$$
s_{i}^{-1}(0)=\sum_{\rho \in \mathcal{B}_{1}(I ; i)} \overline{\mathcal{M}}_{1, \rho}, \quad \text { where } \quad \mathcal{B}_{1}(I ; i)=\left\{\left(I_{P},\left\{I_{B}\right\}\right) \in \mathcal{A}_{1}(I): i \in I_{B}\right\} .
$$

If $I$ and $J$ are finite sets such that $I$ is not empty and $|I|+|J| \geq 2$, then

$$
\mathcal{B}_{1}(I \sqcup J ; i) \subset \mathcal{A}_{1}(I, J) \quad \forall i \in I .
$$

For each $i \in I$, let

$$
L_{0, i}=L_{i} \longrightarrow \overline{\mathcal{M}}_{1,(I, J)}^{0}, \quad s_{0, i}=s_{i} \in H^{0}\left(\overline{\mathcal{M}}_{1,(I, J)}^{0} ; \operatorname{Hom}\left(L_{0, i}, \mathbb{E}^{*}\right)\right)
$$

Suppose $\rho \in \mathcal{A}_{1}(I, J)$ and we have constructed line bundles $L_{\rho-1, i} \longrightarrow \overline{\mathcal{M}}_{1,(I, J)}^{\rho-1}$ for $i \in I$ and bundle sections

$$
s_{\rho-1, i} \in H^{0}\left(\overline{\mathcal{M}}_{1,(I, J)}^{\rho-1} ; \operatorname{Hom}\left(L_{\rho-1, i}, \pi_{\rho-1}^{*} \mathbb{E}^{*}\right)\right)
$$

such that

$$
s_{\rho-1, i}^{-1}(0)=\sum_{\rho^{*} \in \mathcal{B}_{1}(I \sqcup J ; i), \rho^{*}>\rho-1} \overline{\mathcal{M}}_{1, \rho^{*}}^{\rho-1}
$$

\footnotetext{
${ }^{4}$ If $\rho, \rho^{\prime} \in \mathcal{A}_{1}(I, J)$ are not comparable with respect to $\prec$ and $\rho<\rho^{\prime}, \overline{\mathcal{M}}_{1, \rho}^{\rho-1}$ and $\overline{\mathcal{M}}_{1, \rho^{\prime}}^{\rho-1}$ are disjoint

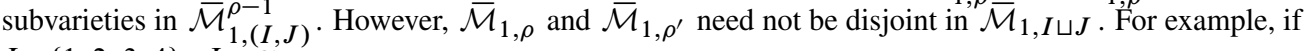
$I=\{1,2,3,4\}, J=\varnothing$,

$$
\rho_{12}=((\{3,4\}),\{\{1,2\}\}), \quad \rho_{34}=((\{1,2\}),\{\{3,4\}\}), \quad \rho_{12,34}=((\varnothing),\{\{1,2\},\{3,4\}\}),
$$

then $\overline{\mathcal{M}}_{1, \rho_{12}}$ and $\overline{\mathcal{M}}_{1, \rho_{34}}$ intersect at $\overline{\mathcal{M}}_{1, \rho_{12,34}}$ in $\overline{\mathcal{M}}_{1,4}$, but their proper transforms in the blowup of $\overline{\mathcal{M}}_{1,4}$ along $\overline{\mathcal{M}}_{1, \rho_{12,34}}$ are disjoint.
} 
By (3-5), this assumption is satisfied for $\rho-1=0$. If

$$
\rho=\left(I_{P} \sqcup J_{P},\left\{I_{k} \sqcup J_{k}: k \in K\right\}\right)
$$

and $i \in I$, we define

$$
L_{\rho, i}= \begin{cases}\tilde{\pi}_{\rho}^{*} L_{\rho-1, i} \otimes \mathcal{O}\left(\overline{\mathcal{M}}_{1, \rho}^{\rho}\right), & \text { if } i \notin I_{P} \\ \tilde{\pi}_{\rho}^{*} L_{\rho-1, i}, & \text { if } i \in I_{P}\end{cases}
$$

By the inductive assumption, $s_{\rho-1, i}$ induces a section $s_{\rho, i}$ of $L_{\rho, i}^{*} \otimes \pi_{\rho}^{*} \mathbb{E}^{*}$ such that

$$
s_{\rho, i}^{-1}(0)=\sum_{\rho^{*} \in \mathcal{B}_{1}(I \sqcup J ; i), \rho^{*}>\rho} \overline{\mathcal{M}}_{1, \rho^{*}}^{\rho}
$$

Thus, the inductive assumption (3-6) is satisfied with $\rho-1$ replaced by $\rho$. Let

$$
\tilde{L}_{i}=L_{\rho_{\max }, i} \longrightarrow \widetilde{\mathcal{M}}_{1,(I, J)}, \quad \tilde{s}_{i}=s_{\rho_{\max }, i} \in H^{0}\left(\widetilde{\mathcal{M}}_{1,(I, J)} ; \operatorname{Hom}\left(\widetilde{L}_{i}, \tilde{\pi}^{*} \mathbb{E}^{*}\right)\right) .
$$

By (3-6), $\tilde{s}_{i}: \tilde{L}_{i} \longrightarrow \tilde{\pi}^{*} \mathbb{E}^{*}$ is an isomorphism of line bundles.

Remark The line bundles $\widetilde{L}_{i}$ and bundle isomorphisms $\tilde{s}_{i}$ just defined are not the same as in Subsection 2.3 in [13] or Subsection 2.1 in [19].

\subsection{Blowups of moduli spaces of genus-zero curves}

In this subsection we give a formula for the numbers (2-7) that involves the blowups of moduli spaces of genus-zero curves defined in Subsection 2.4 of [13] and moduli spaces of genus-one curves, not their blowups. The formula of Proposition 3.1 will be used at the conclusion of Section 3.4.

If $I$ is a finite set, let

$$
\mathcal{A}_{0}(I)=\left\{\left(I_{P},\left\{I_{k}: k \in K\right\}\right): K \neq \varnothing ; I=\bigsqcup_{k \in\{P\} \sqcup K} I_{k} ;\left|I_{k}\right| \geq 2 \forall k \in K ;|K|+\left|I_{P}\right| \geq 2\right\} .
$$

Similarly to Section 3.1, each element $\rho$ of $\mathcal{A}_{0}(I)$ describes a smooth subvariety

$$
\overline{\mathcal{M}}_{0, \rho} \subset \overline{\mathcal{M}}_{0,\{0\} \sqcup I},
$$

with the "principal" component of each curve in $\mathcal{M}_{0, \rho}$ carrying the marked points indexed by the set $\{0\} \sqcup J_{P}$. There is a partial ordering $\prec$ on $\mathcal{A}_{0}(I)$, defined analogously to the partial ordering $\prec$ on $\mathcal{A}_{1}(I)$. If $J$ is also a finite set, let

$$
\mathcal{A}_{0}(I, J)=\left\{\left(\left(I_{P} \sqcup J_{P}\right),\left\{I_{k} \sqcup J_{k}: k \in K\right\}\right) \in \mathcal{A}_{0}(I \sqcup J): I_{k} \neq \varnothing \forall k \in K\right\} .
$$


Suppose $\boldsymbol{\aleph}$ is a finite nonempty set and $\varrho=\left(I_{l}, J_{l}\right)_{l \in \mathbb{N}}$ is a tuple of finite sets such that $I_{l} \neq \varnothing$ and $\left|I_{l}\right|+\left|J_{l}\right| \geq 2$ for all $l \in \aleph$. Let

$$
\overline{\mathcal{M}}_{0, \varrho}=\prod_{l \in \mathcal{N}} \overline{\mathcal{M}}_{0,\{0\} \sqcup I_{l} \sqcup J_{l}} \quad \text { and } \quad F_{\varrho}=\bigoplus_{l \in \mathcal{N}} \pi_{l}^{*} L_{0} \longrightarrow \overline{\mathcal{M}}_{0, \varrho},
$$

where $L_{0} \longrightarrow \overline{\mathcal{M}}_{0,\{0\} \sqcup I_{l} \sqcup J_{l}}$ is the universal tangent line bundle for the marked point 0 and

$$
\pi_{l}: \overline{\mathcal{M}}_{0, \varrho} \longrightarrow \overline{\mathcal{M}}_{0,\{0\} \sqcup I_{l} \sqcup J_{l}}
$$

is the projection map. Denote by

$$
\gamma_{\varrho} \longrightarrow \mathbb{P} F_{\varrho}
$$

the tautological line bundle.

With $\varrho$ as above, let

$$
\begin{aligned}
& \mathcal{A}_{0}(\varrho)=\left\{\left(\boldsymbol{\aleph}_{P},\left(\rho_{l}\right)_{l \in \boldsymbol{N}}\right): \boldsymbol{\aleph}_{P} \subset \boldsymbol{\aleph}, \boldsymbol{\aleph}_{P} \neq \varnothing ;\right. \\
& \rho_{l} \in\left\{\left(I_{l} \sqcup J_{l}, \varnothing\right)\right\} \sqcup \mathcal{A}_{0}\left(I_{l}, J_{l}\right) \forall l \in \boldsymbol{\aleph} ; \\
&\left.\rho_{l}=\left(I_{l} \sqcup J_{l}, \varnothing\right) \forall l \in \boldsymbol{\aleph}-\aleph_{P} ;\left(\boldsymbol{\aleph}_{P},\left(\rho_{l}\right)_{l \in \boldsymbol{N}}\right) \neq\left(\boldsymbol{\aleph},\left(I_{l} \sqcup J_{l}, \varnothing\right)_{l \in \boldsymbol{N}}\right)\right\} .
\end{aligned}
$$

We define a partial ordering on $\mathcal{A}_{0}(\varrho)$ by setting

$$
\rho^{\prime} \equiv\left(\aleph_{P}^{\prime},\left(\rho_{l}^{\prime}\right)_{l \in \mathbb{N}}\right) \prec \rho \equiv\left(\boldsymbol{\aleph}_{P},\left(\rho_{l}\right)_{l \in \boldsymbol{N}}\right)
$$

if $\rho^{\prime} \neq \rho, \aleph_{P}^{\prime} \subset \aleph_{P}$, and for every $l \in \boldsymbol{\aleph}$ either $\rho_{l}^{\prime}=\rho_{l}, \rho_{l}^{\prime} \prec \rho_{l}$, or $\rho_{l}^{\prime}=\left(I_{l} \sqcup J_{l}, \varnothing\right)$. Let $<$ be an ordering on $\mathcal{A}_{0}(\varrho)$ extending the partial ordering $\prec$. Denote the corresponding minimal and maximal elements of $\mathcal{A}_{0}(\varrho)$ by $\rho_{\min }$ and $\rho_{\max }$, respectively. If $\rho \in \mathcal{A}_{0}(\varrho)$, define

$$
\rho-1 \in\{0\} \sqcup \mathcal{A}_{0}(\varrho)
$$

as in (3-3).

If $\rho \in \mathcal{A}_{0}(\varrho)$ is as in $(3-10)$, let

$\overline{\mathcal{M}}_{0, \rho}=\prod_{l \in \mathbb{N}} \overline{\mathcal{M}}_{0, \rho_{l}}, \quad F_{\rho}=\left.\bigoplus_{l \in \mathcal{N}_{P}} \pi_{l}^{*} L_{0}\right|_{\overline{\mathcal{M}}_{0, \rho}} \subset F_{\varrho}, \quad \widetilde{\mathcal{M}}_{0, \rho}^{0}=\mathbb{P} F_{\rho} \subset \widetilde{\mathcal{M}}_{0, \varrho}^{0} \equiv \mathbb{P} F_{\varrho}$.

The spaces $\widetilde{\mathcal{M}}_{0, \varrho}^{0}$ and $\widetilde{\mathcal{M}}_{0, \rho}^{0}$ can be represented by diagrams as in Figure 3. The thinner lines represent typical elements of the spaces $\overline{\mathcal{M}}_{0, \rho_{l}}$, with the marked point 0 lying on the thicker vertical line. We indicate the elements of $\aleph_{P} \subset \aleph$ with the letter $P$ 
next to these points. Note that by (3-9), every dot on a vertical line for which the corresponding tree has more than one line must be labeled with a $P$.
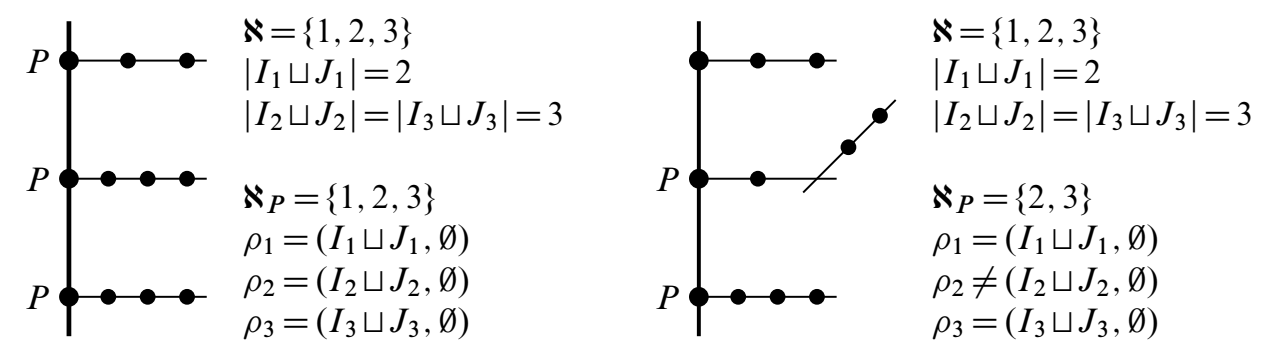

Figure 3: Typical elements of $\widetilde{\mathcal{M}}_{0, \varrho}^{0}$ and $\widetilde{\mathcal{M}}_{0, \rho}$

The blowup construction now proceeds similarly to that in Section 3.1 with

$$
\mathbb{E}_{0}=\gamma_{\varrho} \longrightarrow \widetilde{\mathcal{M}}_{0, \varrho}^{0}
$$

The analogue of (3-4) has the same form:

$$
\mathbb{E}_{\rho}=\tilde{\pi}_{\rho}^{*} \mathbb{E}_{\rho-1} \otimes \mathcal{O}\left(\widetilde{\mathcal{M}}_{0, \rho}^{\rho}\right)
$$

As before, we take

$$
\widetilde{\mathcal{M}}_{0, \varrho}=\widetilde{\mathcal{M}}_{0, \varrho}^{\rho_{\max }}, \quad \tilde{\mathbb{E}}=\mathbb{E}_{\rho_{\max }}, \quad \tilde{\psi}=c_{1}(\tilde{\mathbb{E}}) .
$$

As in Section 3.1, the end result of the above blowup construction is well-defined, ie independent of the choice of the ordering $<$ extending the partial ordering $\prec$.

We now return to the construction of Section 3.1. If $\rho \in \mathcal{A}_{1}(I, J)$ is as in (3-7), let

$$
I_{P}(\rho)=I_{P}, \quad J_{P}(\rho)=J_{P}, \quad \aleph(\rho)=K, \quad \varrho_{B}(\rho)=\left(I_{l}, J_{l}\right)_{l \in \mathbb{N}(\rho)} .
$$

Note that

$$
\overline{\mathcal{M}}_{1, \rho} \approx \overline{\mathcal{M}}_{1, I_{P}(\rho) \sqcup J_{P}(\rho) \sqcup \aleph(\rho)} \times \overline{\mathcal{M}}_{0, \varrho_{B}(\rho)} .
$$

If $\rho=0$, we set

$$
I_{P}(\rho)=I, \quad J_{P}(\rho)=J, \quad \aleph(\rho)=\varnothing, \quad\left\langle\tilde{\psi}^{\tilde{c}},\left[\widetilde{\mathcal{M}}_{0, \varrho_{B}(\rho)}\right]\right\rangle= \begin{cases}1, & \text { if } \tilde{c}=-1 \\ 0, & \text { otherwise }\end{cases}
$$

Let $\lambda=c_{1}(\mathbb{E})$ be the Hodge class on $\overline{\mathcal{M}}_{1, N}$. 
Proposition 3.1 If $I$ and $J$ are finite sets and $\left(\tilde{c},\left(c_{j}\right)_{j \in J}\right) \in \mathbb{Z} \times \mathbb{Z}^{J}$, then

$$
\begin{aligned}
& \left\langle\tilde{c} ;\left(c_{j}\right)_{j \in J}\right\rangle_{(I, J)} \\
& =\sum_{\rho \in\{0\} \sqcup \mathcal{A}_{1}(I, J)}\left(\prod_{j \in J_{P}(\rho)} \psi_{j}^{c_{j}},\left[\overline{\mathcal{M}}_{1, I_{P}(\rho) \sqcup J_{P}(\rho) \sqcup \aleph(\rho)}\right]\right\rangle\left\langle\tilde{\psi}^{\tilde{c}-1} \prod_{j \in J-J_{P}(\rho)} \psi_{j}^{c_{j}},\left[\widetilde{\mathcal{M}}_{0, \varrho_{B}(\rho)}\right]\right\rangle \\
& \left.\quad+\left\langle\lambda \prod_{j \in J_{P}(\rho)} \psi_{j}^{c_{j}},\left[\overline{\mathcal{M}}_{1, I_{P}(\rho) \sqcup J_{P}(\rho) \sqcup \aleph(\rho)}\right]\right\rangle\left\langle\tilde{\psi}^{\tilde{c}-2} \prod_{j \in J-J_{P}(\rho)} \psi_{j}^{c_{j}},\left[\widetilde{\mathcal{M}}_{0, \varrho_{B}(\rho)}\right]\right\rangle\right) .
\end{aligned}
$$

It is immediate that this statement holds if $\tilde{c} \leq 0$. For each $\rho \in \mathcal{A}_{1}(I, J)$, let

$$
\widetilde{\mathcal{M}}_{1, \rho} \subset \widetilde{\mathcal{M}}_{1,(I, J)}
$$

be the proper transform of the exceptional divisor $\overline{\mathcal{M}}_{1, \rho}^{\rho}$ for the blowup at step $\rho$. Since $\overline{\mathcal{M}}_{1, \rho}^{\rho}$ is a divisor in $\overline{\mathcal{M}}_{1,(I, J)}^{\rho}$ and the blowup loci $\overline{\mathcal{M}}_{1, \rho^{*}}^{\rho}$, with $\rho^{*}>\rho$, are not contained in $\overline{\mathcal{M}}_{1, \rho}^{\rho}, \widetilde{\mathcal{M}}_{1, \rho}$ is the pullback of the cohomology class determined by $\overline{\mathcal{M}}_{1, \rho}^{\rho}$ under the blowdown map

$$
\widetilde{\mathcal{M}}_{1,(I, J)} \longrightarrow \overline{\mathcal{M}}_{1,(I, J)}^{\rho} .
$$

Therefore, by (3-4),

$$
\tilde{\psi}=\lambda+\sum_{\rho \in \mathcal{A}_{1}(I, J)} \widetilde{\mathcal{M}}_{1, \rho} \in H^{2}\left(\widetilde{\mathcal{M}}_{1,(I, J)}\right)
$$

Furthermore, by an inductive argument on the stages of the blowup construction similar to Subsections 3.4 and 4.3 in [13],

$$
\widetilde{\mathcal{M}}_{1, \rho} \approx \widetilde{\mathcal{M}}_{1,\left(I_{P}(\rho) \sqcup \aleph(\rho), J_{P}(\rho)\right)} \times \widetilde{\mathcal{M}}_{0, \varrho_{B}(\rho)},\left.\quad \tilde{\psi}\right|_{\widetilde{\mathcal{M}}_{1, \rho}} \approx \pi_{B}^{*} \tilde{\psi},
$$

where $\pi_{B}$ is projection onto the second component. ${ }^{5}$ The $\tilde{c}=1$ case of Proposition 3.1 follows immediately from (3-13) and (3-14). If $\tilde{c} \geq 2$, then by (3-13), (3-14), and

\footnotetext{
${ }^{5}$ The induction begins with (3-12) and $\left.\lambda\right|_{\overline{\mathcal{M}}_{1, \rho}}=\pi_{P}^{*} \lambda$. One then shows that

$$
\overline{\mathcal{M}}_{1, \rho}^{\rho-1} \approx \widetilde{\mathcal{M}}_{1,\left(I_{P}(\rho) \sqcup \aleph(\rho), J_{P}(\rho)\right)} \times \overline{\mathcal{M}}_{0, \varrho_{B}(\rho)},\left.\quad c_{1}\left(\mathbb{E}_{\varrho-1}\right)\right|_{\overline{\mathcal{M}}_{1, \rho}^{\rho-1}}=\pi_{P}^{*} \tilde{\psi}
$$

and the normal bundle of $\overline{\mathcal{M}}_{1, \rho}^{\rho-1}$ is $\pi_{P}^{*} \widetilde{\mathbb{E}}^{*} \otimes \pi_{B}^{*} F_{\varrho_{B}(\rho)}$. Thus,$$
\overline{\mathcal{M}}_{1, \rho}^{\rho} \approx \widetilde{\mathcal{M}}_{1,\left(I_{P}(\rho) \sqcup \aleph(\rho), J_{P}(\rho)\right)} \times \widetilde{\mathcal{M}}_{0, \varrho_{B}(\rho)}^{0},\left.\quad c_{1}\left(\mathbb{E}_{\varrho}\right)\right|_{\overline{\mathcal{M}}_{1, \rho}^{\rho}}=\pi_{B}^{*} c_{1}\left(\mathbb{E}_{0}\right) .
$$

The proper transforms of $\overline{\mathcal{M}}_{1, \rho}^{\rho}$ under blowups along $\overline{\mathcal{M}}_{1, \rho^{*}}^{\rho^{*}-1}$ with $\rho \prec \rho^{*}$ correspond to blowups of the second component of $\overline{\mathcal{M}}_{1, \rho}^{\rho}$ and the twisting (3-11) changes $\pi_{B}^{*} c_{1}\left(\mathbb{E}_{0}\right)$ to $\pi_{B}^{*} c_{1}(\widetilde{\mathbb{E}})$.
} 
$\lambda^{2}=0$, we have

$$
\begin{aligned}
\tilde{\psi} & =\tilde{\psi}^{\tilde{c}-2}\left(\lambda+\sum_{\rho \in \mathcal{A}_{1}(I, J)} \widetilde{\mathcal{M}}_{1, \rho}\right)^{2}=\tilde{\psi}^{\tilde{c}-1} \sum_{\rho \in \mathcal{A}_{1}(I, J)} \widetilde{\mathcal{M}}_{1, \rho}+\lambda \tilde{\psi}^{\tilde{c}-2} \sum_{\rho \in \mathcal{A}_{1}(I, J)} \widetilde{\mathcal{M}}_{1, \rho} \\
& =\sum_{\rho \in \mathcal{A}_{1}(I, J)}\left(\pi_{B}^{*} \tilde{\psi}^{\tilde{c}-1}+\left(\pi_{P}^{*} \lambda\right)\left(\pi_{B}^{*} \tilde{\psi}^{\tilde{c}-2}\right)\right) \widetilde{\mathcal{M}}_{1, \rho} .
\end{aligned}
$$

This implies the $\tilde{c} \geq 2$ cases of Proposition 3.1.

\subsection{Analytic setup}

We now recall the parts of Subsections 1.3, 1.4, 3.1, and 3.2 in [15] needed to formulate Proposition 3.1 of [15] giving a description of the difference between the two genus-one GW-invariants.

An element of the moduli space $\overline{\mathfrak{M}}_{g, k}(X, \beta ; \mathcal{J})$ is represented by a stable continuous degree- $\beta$ map $u$ from a pre-stable genus- $g$ Riemann surface $(\Sigma, j)$ with $k$ marked points to $X$ which is smooth on each component of $\Sigma$ and satisfies the Cauchy-Riemann equation corresponding to $(\mathcal{J}, j)$ :

$$
\bar{\partial}_{\mathcal{J}, j} u \equiv \frac{1}{2}(d u+\mathcal{J} \circ d u \circ j)=0 .
$$

We denote by $\overline{\mathfrak{M}}_{g, k}(X, \beta ; \mathcal{J}, v)$ the space of solutions to the $v$-perturbed CauchyRiemann equation:

$$
\bar{\partial}_{\mathcal{J}, j} u+v(u)=0 .
$$

The perturbation term $v(u)$ is a section of the vector bundle

$$
\Lambda_{\mathcal{J}, j}^{0,1} T^{*} \Sigma \otimes u^{*} T X \equiv\left\{\eta \in \operatorname{Hom}_{\mathbb{R}}\left(T \Sigma, u^{*} T X\right): \mathcal{J} \circ \eta=-\eta \circ j\right\} \longrightarrow \Sigma
$$

and depends continuously on $u$ and smoothly on each stratum $\mathfrak{X}_{\mathcal{T}}(X, \beta)$ of the space $\mathfrak{X}_{g, k}(X, \beta)$ of all continuous degree- $\beta$ maps that are smooth (or $L_{1}^{p}$ with $p>2$ ) on the components of the domain. More formally, $v$ is a multi-section of a Banach orbi-bundle $\Gamma_{g, k}^{0,1}(X, \beta ; \mathcal{J})$ over $\mathfrak{X}_{g, k}(X, \beta){ }^{6}$

\footnotetext{
${ }^{6}$ The topological and analytic aspects of the setup in Subsection 1.3 of [15] are analogous to [3] and [7], respectively.
} 
Suppose $m \in \mathbb{Z}^{+}, J$ is a finite set, and $\beta \in H_{2}(X ; \mathbb{Z})$. If $v$ is a perturbation on the spaces $\mathfrak{X}_{0,\{0\} \sqcup J_{i}}\left(X, \beta_{i}\right)$ as above, with $J_{i} \subset J$ and $\beta_{i} \in H_{2}(X ; \mathbb{Z})$ such that $\omega\left(\beta_{i}\right) \leq \omega(\beta)$, let

$$
\begin{aligned}
& \overline{\mathfrak{M}}_{(m, J)}(X, \beta ; \mathcal{J}, v) \\
&=\left\{\left(b_{i}\right)_{i \in[m]} \in \prod_{i=1}^{i=m} \overline{\mathfrak{M}}_{0,\{0\} \sqcup J_{i}}\left(X, \beta_{i} ; \mathcal{J}, v\right): J_{i} \subset J, \bigsqcup_{i=1}^{i=m} J_{i}=J ;\right. \\
&\left.\beta_{i} \in H_{2}(X ; \mathbb{Z})-\{0\}, \sum_{i=1}^{i=m} \beta_{i}=\beta ; \operatorname{ev}_{0}\left(b_{i}\right)=\operatorname{ev}_{0}\left(b_{i^{\prime}}\right) \forall i, i^{\prime} \in[m]\right\} .
\end{aligned}
$$

Let $\mathfrak{M}_{(m, J)}(X, \beta ; \mathcal{J}, v) \subset \overline{\mathfrak{M}}_{(m, J)}(X, \beta ; \mathcal{J}, v)$ be the subspace consisting of $m$-tuples of maps from smooth domains. Define

$$
\begin{aligned}
& \pi_{i}: \overline{\mathfrak{M}}_{(m, J)}(X, \beta ; \mathcal{J}, v) \longrightarrow \bigsqcup_{\beta_{i} \in H_{2}(X ; \mathbb{Z})-0} \bigsqcup_{J_{i} \subset J} \overline{\mathfrak{M}}_{0,\{0\} \sqcup J_{i}}\left(X, \beta_{i} ; \mathcal{J}, v\right), \\
& \eta_{p}, \tilde{\eta}_{p} \in H^{2 p}\left(\overline{\mathfrak{M}}_{(m, J)}(X, \beta ; \mathcal{J}, v)\right), \quad \operatorname{ev}_{0}: \overline{\mathfrak{M}}_{(m, J)}(X, \beta ; \mathcal{J}) \longrightarrow X,
\end{aligned}
$$

as in Section 2.1.

We will call a perturbation $v$ on $\mathfrak{X}_{0,\{0\} \sqcup J}(X, \beta)$ supported away from $x_{0}$ if $v(u)$ vanishes on a neighborhood of the marked point $x_{0}$ for every element $[\Sigma, u]$ of $\mathfrak{X}_{0,\{0\} \sqcup J}(X, \beta)$. In such a case, $u$ is holomorphic on a neighborhood of the marked point $x_{0}$ for every element $[\Sigma, u]$ of $\overline{\mathfrak{M}}_{0,\{0\} \sqcup J}(X, \beta ; \mathcal{J}, \nu)$. Therefore, there is a well-defined ( $\mathbb{C}$-linear) vector bundle homomorphism $(\mathrm{VBH})$

$$
\mathcal{D}_{0}: L_{0} \longrightarrow \operatorname{ev}_{0}^{*} T X,\left.\quad[\Sigma, u ; w] \longrightarrow d u\right|_{x_{0}} w \text { if }\left.w \in L_{0}\right|_{[\Sigma, u]}=T_{x_{0}} \Sigma,
$$

over $\overline{\mathfrak{M}}_{0,\{0\} \sqcup J}(X, \beta ; \mathcal{J}, v)$. If $m, J$, and $v$ are as in the previous paragraph and $v$ is supported away from $x_{0}$, we obtain $m$ VBHs

$$
\pi_{i}^{*} \mathcal{D}_{0}: \pi_{i}^{*} L_{0} \longrightarrow \operatorname{ev}_{0}^{*} T X
$$

over $\overline{\mathfrak{M}}_{0,(m, J)}(X, \beta ; \mathcal{J}, v)$. The difference between the standard and reduced genusone GW-invariants is described below in terms of these VBHs and the homomorphisms $s_{i}$ defined in Section 3.1.

In the genus-one case, Definition 1.2 in [15] describes a class of perturbations $v$ called effectively supported. These perturbations vanish on all components of the domain of a stable map $u$ on which the degree of $u$ is zero, as well as near such components (including after small deformations of $[\Sigma, u]$ ). If $v_{\mathrm{es}}$ is effectively supported, $[\Sigma, u]$ is an element of $\overline{\mathfrak{M}}_{1, k}\left(X, \beta ; \mathcal{J}, v_{\mathrm{es}}\right)$, and $u$ has degree 0 on a component $\Sigma_{i}$ of $\Sigma$, then $u$ is constant on $\Sigma$. For a generic effectively supported perturbation $v_{\mathrm{es}}$, 
$\overline{\mathfrak{M}}_{1, k}\left(X, \beta ; \mathcal{J}, v_{\mathrm{es}}\right)$ has the same general topological structure as $\overline{\mathfrak{M}}_{1, k}\left(\mathbb{P}^{n}, d\right)$. In particular, if $(X, \omega, \mathcal{J})$ is sufficiently regular (eg a low-degree projective hypersurface), $\nu_{\text {es }}$ can be taken to be 0 for our purposes.

A stratum $\mathfrak{X}_{\mathcal{T}}$ of $\mathfrak{X}_{1, k}(X, \beta)$ is specified by the topological type of the domain $\Sigma$ of the stable maps $u$ in $\mathfrak{X}_{\mathcal{T}}$, including the distribution of the $k$ marked points, and the choice of the components of $\Sigma$ on which the degree of $u$ is not zero. A stratum $\mathfrak{X}_{\mathcal{T}}$ of $\mathfrak{X}_{1, k}(X, \beta)$ will be called degenerate if the degree of any map $u$ in $\mathfrak{X}_{\mathcal{T}}$ on the principal, genus-carrying, component(s) of its domain is zero. The restriction of $u$ to a component $\Sigma_{i} \approx S^{2}$ of $\Sigma$ on which the degree of $u$ is not zero defines a projection

$$
\pi_{\mathcal{T} ; i}: \mathfrak{X}_{\mathcal{T}} \longrightarrow \bigsqcup_{\beta_{i} \in H_{2}(X ; \mathbb{Z})-0} \mathfrak{X}_{0, K_{i}} \sqcup J_{i}\left(X, \beta_{i}\right)
$$

for some $J_{i} \subset[k]$ and finite set $K_{i}$ consisting of the nodes of $\Sigma_{i}$. If $\mathfrak{X}_{\mathcal{T}}$ is degenerate, $K_{i}$ has a distinguished element, the node closest to the principal component(s) of $\Sigma$; it will be denoted by 0 . As in Subsection 3.2 in [15], let $\mathfrak{G}_{1, k}^{\mathrm{gd}}(X, \beta ; \mathcal{J})$ be the space of all effectively supported deformations $v_{\mathrm{es}}$ such that for every degenerate stratum $\mathfrak{X}_{\mathcal{T}}$, $[\Sigma, u] \in \mathfrak{X}_{\mathcal{T}}$, and every component $\Sigma_{i} \approx S^{2}$ of $\Sigma$ on which the degree of $u$ is nonzero

$$
\left.v_{\mathrm{es}}(u)\right|_{\Sigma_{i}}=\left\{\pi_{\mathcal{T} ; i}^{*} \nu_{\mathcal{T} ; i}\right\}(u) \mid \Sigma_{i}
$$

for a fixed (independent of $u$ ) perturbation $v_{\mathcal{T} ; i}$ on the right-hand side of (3-15) such that for every $\beta_{i} \in H_{2}(X ; \mathbb{Z})-0$ with $\omega\left(\beta_{i}\right) \leq \omega(\beta)$ :

(gd1) the linearization

$$
D_{\mathcal{J}, \nu_{\mathcal{T} ; i} ; b}:\left\{\xi \in \Gamma\left(\Sigma_{b} ; u_{b}^{*} T X\right): \xi\left(x_{0}(b)\right)=0\right\} \longrightarrow \Gamma\left(\Sigma_{b} ; \Lambda_{\mathcal{J}, j}^{0,1} T^{*} \Sigma_{b} \otimes u_{b}^{*} T X\right)
$$

of $\bar{\partial}_{\mathcal{J}}+v_{\mathcal{T} ; i}$ at $b$ is surjective for every $b \equiv\left[\Sigma_{b}, u_{b}\right] \in \overline{\mathfrak{M}}_{0, K_{i}} \sqcup J_{i}\left(X, \beta_{i} ; \mathcal{J}, v_{\mathcal{T} ; i}\right)$;

$(\operatorname{gd} 2)$ the restriction of the section

$$
\mathcal{D}_{0} \in \Gamma\left(\overline{\mathfrak{M}}_{0, K_{i}} \sqcup J_{i}\left(X, \beta_{i} ; \mathcal{J}, v_{\mathcal{T} ; i}\right) ; \operatorname{Hom}\left(L_{0}, \mathrm{ev}_{0}^{*} T X\right)\right)
$$

to every stratum of $\overline{\mathfrak{M}}_{0, K_{i} \sqcup J_{i}}\left(X, \beta_{i} ; \mathcal{J}, v_{\mathcal{T} ; i}\right)$ for which the degree of the maps on the component containing $x_{0}$ is nonzero is transverse to the zero section.

If $m \in \mathbb{Z}^{+}$and $J \subset[k]$, let

$$
\mathfrak{M}_{1, k}^{m, J}\left(X, \beta ; \mathcal{J}, v_{\mathrm{es}}\right) \subset \overline{\mathfrak{M}}_{1, k}(X, \beta ; \mathcal{J}, \nu)
$$

be the subspace consisting of the stable maps $[\Sigma, u]$ such that $\Sigma$ is a union of a smooth torus $\Sigma_{P}$ and $m$ spheres attached directly to $\Sigma_{P}$, the degree of $u$ is zero on $\Sigma_{P}$ and 
nonzero on each of the $m$ spheres, and $\Sigma_{\boldsymbol{P}}$ carries the marked points indexed by $J$. If $\nu_{\mathrm{es}} \in \mathfrak{G}_{1, k}^{\mathrm{gd}}(X, \beta ; \mathcal{J})$, there is a natural splitting

$$
\mathfrak{M}_{1, k}^{m, J}\left(X, \beta ; \mathcal{J}, v_{\mathrm{es}}\right) \approx\left(\mathcal{M}_{1,[m] \sqcup J} \times \mathfrak{M}_{(m,[k]-J)}\left(X, \beta ; \mathcal{J}, v_{B}\right)\right) / S_{m},
$$

where $\mathcal{M}_{1,[m] \sqcup J} \subset \overline{\mathcal{M}}_{1,[m] \sqcup J}$ is the subspace of smooth curves and $v_{B}$ is a perturbation supported away from $x_{0}$. With our assumptions on $v_{\mathrm{es}}, v_{B}$ is in fact effectively supported. Furthermore, $\overline{\mathfrak{M}}_{(m,[k]-J)}\left(X, \beta ; \mathcal{J}, v_{B}\right)$ is stratified by smooth orbifolds (in the sense of Remark 1 in Subsection 1.3 in [15]) with the expected normal bundles (ie analogously to $\left.\overline{\mathfrak{M}}_{(m,[k]-J)}\left(\mathbb{P}^{n}, d\right)\right)$. The splitting in (3-18) extends to an immersion over the closures, from the right hand side to the left.

Let $\mu$ be a tuple as in (2-3) and (2-4). As in Subsection 3.1 in [15], choose generic pseudocycle representatives $f_{j}: \bar{Y}_{j} \longrightarrow X$ for the Poincare duals of the cohomology classes $\mu_{j}$ and let

$$
\overline{\mathfrak{M}}_{1, k}\left(X, \beta ; \mathcal{J}, v_{\mathrm{es}} ;\left(f_{j}\right)_{j \in[k]}\right) \subset \overline{\mathfrak{M}}_{1, k}\left(X, \beta ; \mathcal{J}, v_{\mathrm{es}}\right) \times \prod_{j=1}^{j=k} \bar{Y}_{j}
$$

be the preimage of the diagonal $\Delta_{X}^{k} \subset\left(X^{2}\right)^{k}$ under $\prod_{j=1}^{j=k}\left(\mathrm{ev}_{j} \times f_{j}\right)$. Let

$$
\overline{\mathfrak{M}}_{1, k}\left(X, \beta ; \mathcal{J}, v_{\mathrm{es}} ; \mu\right) \subset \overline{\mathfrak{M}}_{1, k}\left(X, \beta ; \mathcal{J}, v_{\mathrm{es}} ;\left(f_{j}\right)_{j \in[k]}\right)
$$

be the zero set of a section $\varphi$ of the vector bundle

$$
V_{\mu} \equiv \bigoplus_{j=1}^{j=k} c_{j} L_{j}^{*} \longrightarrow \overline{\mathfrak{M}}_{1, k}\left(X, \beta ; \mathcal{J}, v_{\mathrm{es}} ;\left(f_{j}\right)_{j \in[k]}\right) .
$$

For good choices of $f_{j}$ and $\varphi$, the splitting (3-18) induces a splitting

$$
\begin{aligned}
\mathfrak{M}_{1, k}^{m, J}\left(X, \beta ; \mathcal{J}, v_{\mathrm{es}} ; \mu\right) & \equiv\left(\mathfrak{M}_{1, k}^{m, J}\left(X, \beta ; \mathcal{J}, v_{\mathrm{es}}\right) \times \prod_{j=1}^{j=k} Y_{j}\right) \cap \overline{\mathfrak{M}}_{1, k}\left(X, \beta ; \mathcal{J}, v_{\mathrm{es}} ; \mu\right) \\
& \approx\left(\mathcal{M}_{1,[m] \sqcup J}(\mu) \times \mathfrak{M}_{(m,[k]-J)}\left(X, \beta ; \mathcal{J}, v_{B} ; \mu\right)\right) / S_{m},
\end{aligned}
$$

for all $m \in \mathbb{Z}^{+}$and $J \subset[k]$. This splitting extends as an immersion over the compactifications.

Denote by

$$
\begin{aligned}
\pi_{P}, \pi_{B}: \overline{\mathcal{M}}_{1,[m] \sqcup J}(\mu) \times \overline{\mathfrak{M}}_{(m,[k]-J)}\left(X, \beta ; \mathcal{J}, v_{B} ; \mu\right) \\
\longrightarrow \overline{\mathcal{M}}_{1,[m] \sqcup J}(\mu) \times \overline{\mathfrak{M}}_{(m,[k]-J)}\left(X, \beta ; \mathcal{J}, v_{B} ; \mu\right)
\end{aligned}
$$


the two component projection maps. With $s_{i}$ as in Section 3.1 and $\mathcal{D}_{0}$ as above, define

$$
\begin{array}{r}
\mathcal{D}_{1, k}^{m, J}: \bigoplus_{i=1}^{i=m} \pi_{P}^{*} L_{i} \otimes \pi_{B}^{*} \pi_{i}^{*} L_{0} \longrightarrow \pi_{P}^{*} \mathbb{E}^{*} \otimes \pi_{B}^{*} \operatorname{ev}_{0}^{*} T X, \\
\left(v_{i} \otimes w_{i}\right)_{i \in[m]} \longrightarrow \sum_{i=1}^{i=m} s_{i}(v) \otimes \mathcal{D}_{0}\left(w_{i}\right) .
\end{array}
$$

This is a VBH over $\overline{\mathcal{M}}_{1,[m] \sqcup J}(\mu) \times \overline{\mathfrak{M}}_{(m,[k]-J)}\left(X, \beta ; \mathcal{J}, v_{B} ; \mu\right)$, which descends to $\overline{\mathfrak{M}}_{1, k}^{m, J}\left(X, \beta ; \mathcal{J}, v_{\mathrm{es}} ; \mu\right)$.

Finally, suppose $\overline{\mathcal{M}}$ is a compact topological space which is a disjoint union of smooth orbifolds, one of which, $\mathcal{M}$, is a dense open subset of $\overline{\mathcal{M}}$, and the real dimensions of all others do not exceed $\operatorname{dim} \mathcal{M}-2$. Let

$$
E, \mathcal{O} \longrightarrow \overline{\mathcal{M}}
$$

be vector orbi-bundles such that the restrictions of $E$ and $\mathcal{O}$ to every stratum of $\overline{\mathcal{M}}$ is smooth and

$$
\operatorname{rk} \mathcal{O}-\operatorname{rk} E=\frac{1}{2} \operatorname{dim}_{\mathbb{R}} \mathcal{M}
$$

If $\alpha \in \Gamma(\overline{\mathcal{M}} ; \operatorname{Hom}(E, \mathcal{O}))$ is a regular section in the sense of Definition 3.9 in [17], then the signed cardinality of the zero set of the affine bundle map

$$
\psi_{\alpha, \bar{v}} \equiv \alpha+\bar{v}: E \longrightarrow \mathcal{O}
$$

is finite and independent of a generic choice of $\bar{v} \in \Gamma(\overline{\mathcal{M}} ; \mathcal{O})$, by Lemma 3.14 in [17]. We denote it by $N(\alpha)$.

Proposition 3.2 Suppose $(X, \omega, \mathcal{J})$ is a compact almost Kahler manifold of real dimension $2 n, k \in \overline{\mathbb{Z}}^{+}, \beta \in H_{2}(X ; \mathbb{Z}), \mu$ is as in (2-3) and (2-4), and $\nu_{\mathrm{es}} \in \mathfrak{G}_{1, k}^{\mathrm{gd}}(X, \beta ; \mathcal{J})$. If the pseudocycle representatives $f_{j}$ and bundle section $\varphi$ are chosen generically, subject to the existence of a splitting (3-19), then

$$
\mathrm{GW}_{1, k}^{\beta}(\mu)-\mathrm{GW}_{1, k}^{\beta ; 0}(\mu)=\sum_{m=1}^{\infty} \sum_{J \subset[k]} \mathcal{C}_{1, k}^{m, J}(\bar{\partial}),
$$

where $\mathcal{C}_{1, k}^{m, J}(\bar{\partial})$ is the $\bar{\partial}$-contribution of $\mathfrak{M}_{1, k}^{m, J}\left(X, \beta ; \mathcal{J}, v_{\mathrm{es}} ; \mu\right)$ to $\mathrm{GW}_{1, k}^{\beta}(\mu)$. It is given by

$$
\mathcal{C}_{1, k}^{m, J}(\bar{\partial})=N\left(\mathcal{D}_{1, k}^{m, J}\right)
$$


where we view $\mathcal{D}_{1, k}^{m, J}$ as a vector bundle homomorphism over $\overline{\mathfrak{M}}_{1, k}^{m, J}\left(X, \beta ; \mathcal{J}, v_{\mathrm{es}} ; \mu\right)$. In particular, the bundle homomorphism $\mathcal{D}_{1, k}^{m, J}$ is regular.

This is the essence of Proposition 3.1 in [15]. While Subsections 3.1 and 3.2 in [15] explicitly treat only the case without descendants, ie $c_{j}=0$ for all $j \in[k]$, exactly the same argument applies in the general case. The notion of $\bar{\partial}$-contribution of a stratum to $\mathrm{GW}_{1, k}^{\beta}(\mu)$ is made precise in Proposition 3.1 in [15], but (3-20) and (3-21) suffice for our purposes.

Proposition 3.2 shows only the main strata $\mathfrak{M}_{1, k}^{m, J}\left(X, \beta ; \mathcal{J}, v_{\mathrm{es}}\right)$ of $\overline{\mathfrak{M}}_{1, k}\left(X, \beta ; \mathcal{J}, v_{\mathrm{es}}\right)$ contribute to the difference between the standard and reduced genus-one $\mathrm{GW}$-invariants. Furthermore, if $c_{j}=0$ for all $j \in[k]$, ie there are no $\psi$-classes involved, the numbers $\langle\mu\rangle_{1,[m] \sqcup J}$ and $\langle\lambda ; \mu\rangle_{1,[m] \sqcup J}$ in (3-31) below are integrals of 1 and $\lambda$ on $\overline{\mathcal{M}}_{1,[m] \sqcup J}$. Thus, if $c_{j}=0$ for all $j \in[k]$, the contribution of $\mathfrak{M}_{1, k}^{m, J}\left(X, \beta ; \mathcal{J}, v_{\mathrm{es}}\right)$ is zero unless $(m, J)=(1, \varnothing)$. Even in this case, the difference between the two invariants involves genus-zero GW-invariants arising from the closure of the second component in the decomposition (3-18) with $(m, J) \neq(1, \varnothing)$; see $(2-15)$. However, this is also the second component in the analogous decomposition of the boundary stratum

$$
\mathfrak{M}_{(m ; J,[k]-J)}\left(X, \beta ; \mathcal{J}, v_{B}\right) \subset \overline{\mathfrak{M}}_{1, k+1}\left(X, \beta ; \mathcal{J}, v_{B}\right)
$$

defined in Section 3.4. As indicated by the $(m, J)=(1, \varnothing)$ case of (3-31) and its proof, the latter is the reason that the genus-zero $(m, J)$-invariants arise in (2-15).

\subsection{Topological computations}

In this subsection we express the numbers (3-21) in terms of cohomology classes and $\mathrm{GW}$-invariants and thus conclude the proof of Theorem 1A.

With notation as in the previous subsection, let

$$
\overline{\mathfrak{M}}_{(m,[k]-J)} \equiv \overline{\mathfrak{M}}_{(m,[k]-J)}\left(X, \beta ; \mathcal{J}, v_{B} ; \mu\right) .
$$

Equation (3-21) can be restated as

$$
\mathcal{C}_{1, k}^{m, J}(\bar{\partial})=N\left(\mathcal{D}_{1, k}^{m, J}\right) / m !
$$

with $\mathcal{D}_{1, k}^{m, J}$ viewed as a VBH over $\overline{\mathcal{M}}_{1,[m] \sqcup J}(\mu) \times \overline{\mathfrak{M}}_{(m,[k]-J)}$. It is straightforward to see that

$$
N\left(\mathcal{D}_{1, k}^{m, J}\right)=N\left(\widetilde{\mathcal{D}}_{1, k}^{m, J}\right)
$$


where $\widetilde{\mathcal{D}}_{1, k}^{m, J}$ is the VBH over $\widetilde{\mathcal{M}}_{1,([m], J)}(\mu) \times \overline{\mathfrak{M}}_{(m,[k]-J)}$ given by

$$
\begin{array}{r}
\widetilde{\mathcal{D}}_{1, k}^{m, J}: \bigoplus_{i=1}^{i=m} \pi_{P}^{*} \widetilde{L}_{i} \otimes \pi_{B}^{*} \pi_{i}^{*} L_{0} \longrightarrow \pi_{P}^{*} \mathbb{E}^{*} \otimes \pi_{B}^{*} \operatorname{ev}_{0}^{*} T X, \\
\left(v_{i} \otimes w_{i}\right)_{i \in[m]} \longrightarrow \sum_{i=1}^{i=m} \widetilde{s}_{i}(v) \otimes \mathcal{D}_{0}\left(w_{i}\right),
\end{array}
$$

with $\widetilde{L}_{i}$ and $\widetilde{s}_{i}$ as at the end of Section 3.1.

Since $\tilde{s}_{i}: \widetilde{L}_{i} \longrightarrow \mathbb{E}^{*}$ is an isomorphism for all $i \in[m]$,

$$
N\left(\mathcal{D}_{1, k}^{m, J}\right)=N\left(\pi_{P}^{*} \operatorname{id}_{\mathbb{E}^{*}} \otimes \pi_{B}^{*} \mathcal{D}_{(m,[k]-J)}\right),
$$

where $\operatorname{id}_{\mathbb{E}^{*}}$ is viewed as a VBH on $\widetilde{\mathcal{M}}_{1,([m], J)}(\mu)$ and $\mathcal{D}_{(m,[k]-J)}$ is the VBH on $\overline{\mathfrak{M}}_{(m,[k]-J)}$ defined by

$$
\mathcal{D}_{(m,[k]-J)}: F_{(m,[k]-J)} \equiv \bigoplus_{i=1}^{i=m} \pi_{i}^{*} L_{0} \longrightarrow \mathrm{ev}_{0}^{*} T X, \quad\left(w_{i}\right)_{i \in[m]} \longrightarrow \sum_{i=1}^{i=m} \mathcal{D}_{0}\left(w_{i}\right)
$$

This VBH induces a VBH over the projectivization of $F_{(m,[k]-J)}$,

$$
\widetilde{\mathcal{D}}_{0} \in \Gamma\left(\widetilde{\mathfrak{M}}_{(m,[k]-J)}^{0} ; \operatorname{Hom}\left(\mathbb{E}_{0} ; \mathrm{ev}_{0}^{*} T X\right)\right),
$$

where

$$
\widetilde{\mathfrak{M}}_{(m,[k]-J)}^{0}=\mathbb{P} F_{(m,[k]-J)}, \quad \mathbb{E}_{0}=\gamma_{(m,[k]-J)},
$$

and $\gamma_{(m,[k]-J)} \longrightarrow \mathbb{P} F_{(m,[k]-J)}$ is the tautological line bundle. It is straightforward to see from the definition that

$$
N\left(\pi_{P}^{*} \operatorname{id}_{\mathbb{E}^{*}} \otimes \pi_{B}^{*} \mathcal{D}_{(m,[k]-J)}\right)=N\left(\pi_{P}^{*} \operatorname{id}_{\mathbb{E}^{*}} \otimes \pi_{B}^{*} \widetilde{\mathcal{D}}_{0}\right) .
$$

By (gd1) and a dimension count as below, $\mathbb{P} F_{(m,[k]-J)}$ is stratified by orbifolds with the expected normal bundles and $\widetilde{\mathcal{D}}_{0}$ does not vanish on every stratum of $\mathbb{P} F_{(m,[k]-J)}$ on which its restriction is transverse to the zero set (unless $\widetilde{\mathcal{M}}_{1,([m], J)}(\mu)$ is empty). Using (gd2), the strata of $\mathbb{P} F_{(m,[k]-J)}$ on which $\widetilde{\mathcal{D}}_{0}$ is not transverse to the zero set can be described as follows.

\footnotetext{
${ }^{7} \overline{\mathcal{M}}_{1,[m] \sqcup J}(\mu)$ is the zero of a section $\varphi_{J}$ of the vector bundle $V_{\mu ; J} \equiv \bigoplus_{j \in J} c_{j} \widetilde{\mathcal{M}}_{j}^{*}$ over $\overline{\mathcal{M}}_{1,[m] \sqcup J}$ such that the restriction of $\varphi_{J}$ to every blowup locus is transverse to the zero set. $\widetilde{\mathcal{M}}_{1,([m], J)}(\mu)$ is the preimage of $\overline{\mathcal{M}}_{1,[m] \sqcup J}(\mu)$ under the blowdown map $\widetilde{\mathcal{M}}_{1,([m], J)} \longrightarrow \overline{\mathcal{M}}_{1,[m] \sqcup J}$.
} 
If $J$ is a finite set and $\beta \in H_{2}(X ; \mathbb{Z})$, let

$$
\begin{gathered}
\mathcal{A}_{0}(J)=\left\{\left(m ; J_{P}, J_{B}\right): m \in \mathbb{Z}^{+} ; J_{P}, J_{B} \subset J ; m+\left|J_{P}\right| \geq 2\right\} ; \\
\overline{\mathfrak{M}}_{(0, J)}\left(X, \beta ; \mathcal{J}, v_{B}\right)=\overline{\mathfrak{M}}_{0,\{0\} \sqcup J}\left(X, \beta ; \mathcal{J}, v_{B}\right) .
\end{gathered}
$$

If $\sigma=\left(m ; J_{P}, J_{B}\right)$ is an element of $\mathcal{A}_{0}(J)$, let

$$
\mathfrak{M}_{\sigma}\left(X, \beta ; \mathcal{J}, v_{B}\right) \subset \overline{\mathfrak{M}}_{0,\{0\} \sqcup J}\left(X, \beta ; \mathcal{J}, v_{B}\right)
$$

be the subset of consisting of the stable maps $[\Sigma, u]$ such that

(i) the components of $\Sigma$ are $\Sigma_{i}=\mathbb{P}^{1}$ with $i \in\{P\} \sqcup[k]$;

(ii) $\left.u\right|_{\Sigma_{P}}$ is constant and the marked points on $\Sigma_{P}$ are indexed by the set $\{0\} \sqcup J_{P}$;

(iii) for each $i \in[m], \Sigma_{i}$ is attached to $\Sigma_{P}$ and $\left.u\right|_{\Sigma_{i}}$ is not constant.

We denote by

$$
\overline{\mathfrak{M}}_{\sigma}\left(X, \beta ; \mathcal{J}, v_{B}\right) \subset \overline{\mathfrak{M}}_{0,\{0\} \sqcup J}\left(X, \beta ; \mathcal{J}, v_{B}\right)
$$

the closure of $\mathfrak{M}_{\sigma}\left(X, \beta ; \mathcal{J}, v_{B}\right)$. In each diagram of Figure 4 , the irreducible components of $\Sigma$ are represented by lines, and the homology class next to each component shows the degree of $u$ on that component. We indicate the marked points lying on the component $\Sigma_{P}$ only.

If $m \in \mathbb{Z}^{+}$and $J$ is a finite set, let

$$
\begin{array}{r}
\mathcal{A}_{0}(m ; J)=\left\{\left(\left(\sigma_{i}\right)_{i \in[m]}, J_{B}\right):\left(\sigma_{i}, \varnothing\right) \in\{(0, \varnothing)\} \sqcup \mathcal{A}_{0}\left(J_{i, P}\right),\right. \\
\left(\sigma_{i}\right)_{i \in[m]} \neq(0)_{i \in[m]} ; \\
\left.\bigsqcup_{i=1}^{i=m} J_{i, P}=J-J_{B}\right\} .
\end{array}
$$

If $\varrho \equiv\left(\left(\sigma_{i}\right)_{i \in[m]}, J_{B}\right)$ is an element of $\mathcal{A}_{0}(m ; J)$, we put

$$
\aleph_{P}(\varrho)=\left\{i \in[m]: \sigma_{i} \neq 0\right\} \quad \text { and } \quad \aleph_{S}(\varrho)=\left\{i \in[m]: \sigma_{i}=0\right\} .
$$

Here $P$ and $S$ stand for the subsets of principal and secondary elements of $[m]$, respectively. Note that $\aleph_{P}(\varrho) \neq \varnothing$ for all $\varrho \in \mathcal{A}_{0}(m ; J)$. Let

$$
\begin{aligned}
\overline{\mathfrak{M}}_{\varrho}\left(X, \beta ; \mathcal{J}, v_{B}\right)=\left\{\left(b_{i}\right)_{i \in[m]} \in \prod_{i=1}^{i=m} \overline{\mathfrak{M}}_{\left(\sigma_{i}, J_{i, B}\right)}\left(X, \beta_{i} ; \mathcal{J}, v_{B}\right):\right. \\
\left.\sum_{i=1}^{i=m} \beta_{i}=\beta ; \bigsqcup_{i=1}^{i=m} J_{i, B}=J_{B} ; \operatorname{ev}_{0}\left(b_{i_{1}}\right)=\operatorname{ev}_{0}\left(b_{i_{2}}\right) \forall i_{1}, i_{2} \in[m]\right\} .
\end{aligned}
$$

This is a subspace of $\overline{\mathfrak{M}}_{(m, J)}\left(X, \beta ; \mathcal{J}, v_{B}\right)$. 

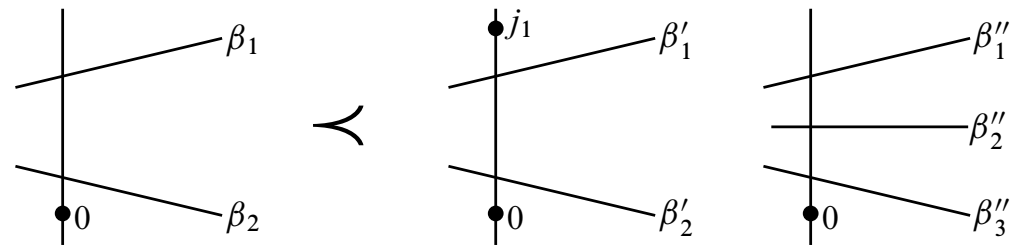

$$
\begin{aligned}
& m=2, J_{P}=\left\{j_{1}\right\} \\
& \beta_{1}^{\prime}, \beta_{2}^{\prime} \neq 0, \beta_{1}^{\prime}+\beta_{2}^{\prime}=\beta
\end{aligned}
$$

Figure 4: Examples of partial ordering (3-24)

With $\mu$ as before and $\varrho \in \mathcal{A}_{0}(m ;[k]-J)$, let

$$
\overline{\mathfrak{M}}_{\varrho}=\left(\overline{\mathfrak{M}}_{\varrho}\left(X, \beta ; \mathcal{J}, v_{B}\right) \times \prod_{j=1}^{j=k} \bar{Y}_{j}\right) \cap \overline{\mathfrak{M}}_{(m,[k]-J)}\left(X, \beta ; \mathcal{J}, v_{B} ; \mu\right) .
$$

Define

$$
F_{\varrho ; P}=\left.\left.\bigoplus_{i \in \mathbb{N}_{P}(\varrho)} \pi_{i}^{*} L_{0}\right|_{\overline{\mathfrak{M}}_{Q}} \subset F_{(m,[k]-J)}\right|_{\overline{\mathfrak{M}}_{\varrho},}, \quad \widetilde{\mathfrak{M}}_{\varrho}^{0}=\mathbb{P} F_{\varrho ; P} \subset \widetilde{\mathfrak{M}}_{(m,[k]-J)}^{0}
$$

It is immediate from the definition of $\widetilde{\mathcal{D}}_{0}$ that it vanishes identically on $\widetilde{\mathfrak{M}}_{\varrho}^{0}$ for every element $\varrho$ in $\mathcal{A}_{0}(m ;[k]-J)$, since $\mathcal{D}_{0}$ vanishes identically on the strata of $\overline{\mathfrak{M}}_{0,\{0\} \sqcup J_{i}}\left(X, \beta_{i} ; \mathcal{J}, v_{B}\right)$ for which the degree of the maps on the component carrying the 0 -th marked point is zero. On the other hand, by $(\mathrm{gd} 2)$, the restriction of $\widetilde{\mathcal{D}}_{0}$ to any stratum of $\widetilde{\mathfrak{M}}_{(m,[k]-J)}^{0}$ in the complement of every $\widetilde{\mathfrak{M}}_{\varrho}^{0}$ is transverse to the zero set and thus does not vanish by a dimension count as below (unless $\widetilde{\mathcal{M}}_{1,([m], J)}(\mu)$ is empty).

As described in Section 3 of [17], the number $N\left(\widetilde{\mathcal{D}}_{0}\right)$ is the euler class of the quotient of the target bundle of $\widetilde{\mathcal{D}}_{0}$ by the domain line bundle minus a correction from $\widetilde{\mathcal{D}}_{0}^{-1}(0)$. The correction splits into contributions from the strata of $\widetilde{\mathcal{D}}_{0}^{-1}(0)$ each of which is again the number of zeros of an affine bundle map, but with the rank of the target bundle reduced by at least one. The linear part of each affine bundle map is determined by the topological behavior of $\widetilde{\mathcal{D}}_{0}^{-1}$ in the normal direction to each stratum. This behavior (for $\mathcal{D}_{0}$ and thus for $\widetilde{\mathcal{D}}_{0}$ ) is described in Subsection 2.4 of [15]. Thus, by iteration, one obtains a finite tree of cohomology classes at the nodes which sum up to $N\left(\widetilde{\mathcal{D}}_{0}\right)$. The tree in this case is similar to a subtree of the tree in Subsection 3.2 of [18], but twisted with $\mathbb{E}^{*}$. Thus, $N\left(\widetilde{\mathcal{D}}_{0}\right)$ can be expressed in terms of cohomology classes by a direct, though laborious, computation nearly identical to the one in Subsections 3.1 and 3.2 in [18]. This time, we will instead compute $N\left(\widetilde{\mathcal{D}}_{0}\right)$ by blowing up $\widetilde{\mathfrak{M}}_{(m,[k]-J)}^{0}$ and 
twisting $\widetilde{\mathcal{D}}_{0}$ to a nowhere-vanishing vector bundle homomorphism $\widetilde{\mathcal{D}}_{(m,[k]-J)}$. This construction is a direct generalization of Section 3 in [13].

Define a partial ordering on the set $\mathcal{A}_{0}(J)$ by setting

$$
\sigma^{\prime} \equiv\left(m^{\prime} ; J_{P}^{\prime}, J_{B}^{\prime}\right) \prec \sigma \equiv\left(m ; J_{P}, J_{B}\right) \quad \text { if } \quad \sigma^{\prime} \neq \sigma, m^{\prime} \leq m, J_{P}^{\prime} \subset J_{P} .
$$

Similarly to Section 3.1, this condition means that the elements of $\mathfrak{M}_{\sigma^{\prime}}(X, \beta ; \mathcal{J}, v)$ can be obtained from the elements of $\mathfrak{M}_{\sigma}\left(X, \beta ; \mathcal{J}, v_{B}\right)$ by moving more points onto the bubble components or combining the bubble components; see Figure 4. The bubble components are the components not containing the marked point 0 . Define a partial ordering $\prec$ on $\mathcal{A}_{0}(m ; J)$ by setting

$$
\varrho^{\prime} \equiv\left(\left(\sigma_{i}^{\prime}\right)_{i \in[m]}, J_{B}^{\prime}\right) \prec \varrho \equiv\left(\left(\sigma_{i}\right)_{i \in[m]}, J_{B}\right)
$$

if $\varrho^{\prime} \neq \varrho$ and for every $i \in[m]$ either $\sigma_{i}^{\prime}=\sigma_{i},\left(\sigma_{i}^{\prime}, \varnothing\right) \prec\left(\sigma_{i}, \varnothing\right)$, or $\sigma_{i}^{\prime}=0$. Note that

$$
\varrho^{\prime} \prec \varrho \Longrightarrow \aleph_{P}\left(\varrho^{\prime}\right) \subset \aleph_{P}(\varrho) ; \quad \varrho=\left(\left(m_{i} ; J_{i, P}\right)_{i \in \aleph_{P}(\varrho)},(0)_{i \in \aleph_{S}(\varrho)}, J_{B}\right)
$$

for some $m_{i}$ and $J_{i, P}$. Choose an ordering $<$ on $\mathcal{A}_{0}(m ; J)$ extending the partial ordering $\prec$. Denote the corresponding minimal element by $\varrho_{\min }$ and the largest element for which $\overline{\mathfrak{M}}_{\varrho}$ is nonempty by $\varrho_{\max }$. For every $\varrho \in \mathcal{A}_{0}(m ; J)$, define

$$
\varrho-1 \in\{0\} \sqcup \mathcal{A}_{0}(m ; J)
$$

as in (3-3).

With $\varrho$ as (3-26), let

$$
\begin{aligned}
\varrho P & =\left(\left[m_{i}\right], J_{i, P}\right)_{i \in \boldsymbol{\aleph}_{P}(\varrho)}, & m_{B}(\varrho) & =\left|\boldsymbol{\aleph}_{S}(\varrho)\right|+\sum_{i \in \boldsymbol{\aleph}_{P}(\varrho)} m_{i}, \\
J_{B}(\varrho) & =J_{B}, & G_{\varrho} & =\prod_{i \in \boldsymbol{\aleph}_{P}(\varrho)} S_{m_{i}} .
\end{aligned}
$$

With $\widetilde{\mathcal{M}}_{0, \varrho_{P}}^{0}$ as in Section 3.2, there is a natural node-identifying immersion

$$
\iota_{0, \varrho}: \widetilde{\mathcal{M}}_{0, \varrho_{P}}^{0}(\mu) \times \overline{\mathfrak{M}}_{\left(m_{B}(\varrho), J_{B}(\varrho)\right)}^{\longrightarrow} \widetilde{\mathfrak{M}}_{\varrho}^{0} \subset \widetilde{\mathfrak{M}}_{(m,[k]-J)}^{0} .
$$

It descends to an immersion

$$
\bar{\iota}_{0, \varrho}:\left(\widetilde{\mathcal{M}}_{0, \varrho_{P}}^{0}(\mu) \times \overline{\mathfrak{M}}_{\left(m_{B}(\varrho), J_{B}(\varrho)\right)}\right) / G_{\varrho} \longrightarrow \widetilde{\mathfrak{M}}_{(m,[k]-J)}^{0},
$$

which is an embedding outside the preimages of $\widetilde{\mathfrak{M}}_{\varrho^{\prime}}^{0}$ with $\varrho^{\prime} \prec \varrho$.

As in the blowup construction of Section 3.1, we inductively define

$$
\tilde{\pi}_{\varrho}: \widetilde{\mathfrak{M}}_{(m,[k]-J)}^{\varrho} \longrightarrow \widetilde{\mathfrak{M}}_{(m,[k]-J)}^{\varrho-1}
$$


to be the blowup of $\widetilde{\mathfrak{M}}_{(m,[k]-J)}^{\varrho-1}$ along the proper transform $\widetilde{\mathfrak{M}}_{\varrho}^{\varrho-1}$ of $\widetilde{\mathfrak{M}}_{\varrho}^{0}$ in $\widetilde{\mathfrak{M}}_{(m,[k]-J)}^{\varrho-1}$. If $\widetilde{\mathfrak{M}}_{\varrho}^{\varrho} \subset \mathfrak{M}_{(m,[k]-J)}^{\varrho}$ is the exceptional divisor, let

$$
\mathbb{E}_{\varrho}=\tilde{\pi}_{\varrho}^{*} \mathbb{E}_{\varrho-1} \otimes \mathcal{O}\left(\widetilde{\mathfrak{M}}_{\varrho}^{\varrho}\right)
$$

The vector bundle homomorphism $\widetilde{\mathcal{D}}_{\varrho-1}: \mathbb{E}_{\varrho-1} \longrightarrow \mathrm{ev}_{0}^{*} T X$ induces a section

$$
\widetilde{\mathcal{D}}_{\varrho} \in \Gamma\left(\widetilde{\mathfrak{M}}_{(m,[k]-J)}^{\varrho} ; \operatorname{Hom}\left(\mathbb{E}_{\varrho}, \mathrm{ev}_{0}^{*} T X\right)\right) .
$$

As described in detail in Subsection 3.4 in [13] (in the case $\left.(X, \mathcal{J})=\mathbb{P}^{n}\right), \iota_{0, \varrho}$ induces an immersion

$$
\iota_{\varrho-1, \varrho}: \widetilde{\mathcal{M}}_{0, \varrho_{P}}(\mu) \times \overline{\mathfrak{M}}_{\left(m_{B}(\varrho), J_{B}(\varrho)\right)} \longrightarrow \widetilde{\mathfrak{M}}_{\varrho}^{\varrho-1} \subset \widetilde{\mathfrak{M}}_{(m,[k]-J)}^{\varrho-1}
$$

and an embedding

$$
\bar{\iota}_{\varrho-1, \varrho}:\left(\widetilde{\mathcal{M}}_{0, \varrho P}(\mu) \times \overline{\mathfrak{M}}_{\left(m_{B}(\varrho), J_{B}(\varrho)\right)}\right) / G_{\varrho} \longrightarrow \widetilde{\mathfrak{M}}_{(m,[k]-J)}^{\varrho-1} .
$$

Thus, the centers of all blowups are smooth (in the appropriate sense) and

$$
\widetilde{\mathfrak{M}}_{\varrho}^{\varrho} \approx\left(\widetilde{\mathcal{M}}_{0, \varrho P}(\mu) \times \widetilde{\mathfrak{M}}_{\left(m_{B}(\varrho), J_{B}(\varrho)\right)}^{0}\right) / G_{\varrho} .
$$

Furthermore,

$$
\left.\tilde{\pi}_{\varrho}^{*} c_{1}\left(\mathbb{E}_{\varrho-1}\right)\right|_{\widetilde{\mathfrak{M}}_{\varrho}^{\varrho}}=\pi_{P}^{*} \tilde{\psi},\left.\quad c_{1}\left(\mathbb{E}_{\varrho}\right)\right|_{\widetilde{\mathfrak{M}}_{\varrho}^{\varrho}}=\pi_{B}^{*} c_{1}\left(\gamma_{\left(m_{B}(\varrho), J_{B}(\varrho)\right)}\right),
$$

where

$$
\pi_{P}, \pi_{B}: \widetilde{\mathcal{M}}_{0, \varrho_{P}}(\mu) \times \widetilde{\mathfrak{M}}_{\left(m_{B}(\varrho), J_{B}(\varrho)\right)}^{0} \longrightarrow \widetilde{\mathcal{M}}_{0, \varrho_{P}}(\mu), \widetilde{\mathfrak{M}}_{\left(m_{B}(\varrho), J_{B}(\varrho)\right)}^{0}
$$

are the projection maps. Finally, the restriction of $\mathcal{D}_{\varrho}$ to every stratum of $\widetilde{\mathfrak{M}}_{(m,[k]-J)}^{\varrho}$ not contained in the proper transform $\widetilde{\mathfrak{M}}_{\varrho^{*}}^{\varrho}$ of $\widetilde{\mathfrak{M}}_{\varrho}^{0}$ for any $\varrho^{*} \in \mathcal{A}_{0}(m ;[k]-J)$ with $\varrho^{*}>\varrho$ is transverse to the zero set. ${ }^{8}$

$$
\begin{array}{cc}
\text { Define } \quad \widetilde{\mathfrak{M}}_{(m,[k]-J)}=\widetilde{\mathfrak{M}}_{(m,[k]-J)}^{\varrho_{\max }, \quad} \quad \widetilde{\mathbb{E}}=\mathbb{E}_{\varrho_{\text {max }},}, \\
\widetilde{\mathcal{D}}_{(m,[k]-J)}=\widetilde{\mathcal{D}}_{\varrho_{\max }} \in \Gamma\left(\widetilde{\mathfrak{M}}_{(m,[k]-J)} ; \operatorname{Hom}\left(\widetilde{\mathbb{E}}, \mathrm{ev}_{0}^{*} T X\right)\right) .
\end{array}
$$

As can be seen directly from the definition,

$$
N\left(\pi_{P}^{*} \mathrm{id}_{\mathbb{E}^{*}} \otimes \pi_{B}^{*} \widetilde{\mathcal{D}}_{0}\right)=N\left(\pi_{P}^{*} \operatorname{id}_{\mathbb{E}^{*}} \otimes \pi_{B}^{*} \widetilde{\mathcal{D}}_{(m,[k]-J)}\right),
$$

\footnotetext{
${ }^{8}$ This statement is obtained as in Subsection 3.4 in [13], using the description of the behavior of $\mathcal{D}_{0}$ in Subsection 2.4 in [15] and the assumption $(\mathrm{gd} 2)$.
}

Geometry $\&$ Topology, Volume 12 (2008) 
where the maps $\pi_{P}$ and $\pi_{B}$ on the right-hand side are the two component projections

$$
\widetilde{\mathcal{M}}_{1,([m], J)}(\mu) \times \widetilde{\mathfrak{M}}_{(m,[k]-J)} \longrightarrow \widetilde{\mathcal{M}}_{1,([m], J)}(\mu), \widetilde{\mathfrak{M}}_{(m,[k]-J)} .
$$

On the other hand, by the previous paragraph, the restriction of $\widetilde{\mathcal{D}}_{(m,[k]-J)}$ to every stratum of $\widetilde{\mathfrak{M}}_{(m,[k]-J)}$ is transverse to the zero set. By (2-1), (2-2), (2-4), and (gd1),

$\operatorname{dim} \widetilde{\mathcal{M}}_{1,([m], J)}(\mu) \times \widetilde{\mathfrak{M}}_{(m,[k]-J)}=2(n-m)+2(m-1)<2 \operatorname{rk}_{\mathbb{C}}\left(\pi_{P}^{*} \mathbb{E}^{*} \otimes \pi_{B}^{*} \operatorname{ev}_{0}^{*} T X\right)$.

Therefore, $\pi_{P}^{*} \operatorname{id}_{\mathbb{E}^{*}} \otimes \pi_{B}^{*} \widetilde{\mathcal{D}}_{(m,[k]-J)}$ is injective and thus

$$
\begin{aligned}
\mathcal{C}_{1, k}^{m, J}(\bar{\partial})= & \frac{1}{m !} N\left(\pi_{P}^{*} \operatorname{id}_{\mathbb{E}^{*}} \otimes \pi_{B}^{*} \widetilde{\mathcal{D}}_{(m,[k]-J)}\right) \\
= & \frac{1}{m !}\left\langle e\left(\left(\pi_{P}^{*} \mathbb{E}^{*} \otimes \pi_{B}^{*} \operatorname{ev}_{0}^{*} T X\right) /\left(\pi_{P}^{*} \mathbb{E}^{*} \otimes \pi_{B}^{*} \widetilde{\mathbb{E}}\right)\right),\right. \\
& {\left[\widetilde{\mathcal{M}}_{1,([m], J)}(\mu) \times \widetilde{\mathfrak{M}}_{(m,[k]-J)]\rangle .}\right.}
\end{aligned}
$$

Remark Since $c_{1}\left(\mathbb{E}^{*}\right)^{2}=\lambda^{2}=0$, the last expression in (3-29) is zero if $m+|J|>1$ and $c_{j}=0$ for all $j \in J$. Thus, if $\mu$ involves no descendants, ie $c_{j}=0$ for all $j \in[k]$, the only stratum of $\overline{\mathfrak{M}}_{1, k}\left(X, \beta ; \mathcal{J}, v_{\mathrm{es}}\right)$ contributing to the difference between the standard and reduced genus-one degree- $\beta$ invariants corresponding to $\mu$ is $\mathfrak{M}_{1, k}^{1, \varnothing}\left(X, \beta ; \mathcal{J}, \nu_{\mathrm{es}}\right)$.

It remains to express the right-hand side of (3-29) in terms of GW-invariants. Let

$$
\begin{gathered}
\langle\mu\rangle_{1,[m] \sqcup J}=\left\langle\prod_{j \in J} \psi_{j}^{c_{j}},\left[\overline{\mathcal{M}}_{1,[m] \sqcup J]}\right], \quad\langle\lambda ; \mu\rangle_{1,[m] \sqcup J}=\left\langle\lambda \prod_{j \in J} \psi_{j}^{c_{j}},\left[\overline{\mathcal{M}}_{1,[m] \sqcup J]}\right],\right.\right. \\
\left\langle\tilde{\psi}^{p} ; \mu\right\rangle_{\left(0, \varrho_{P}\right)}=\left\langle\tilde{\psi}^{p} \prod_{j \in J_{P}(\varrho)} \psi_{j}^{c_{j}},\left[\widetilde{\mathcal{M}}_{0, \varrho_{P}}\right]\right\rangle, \\
\overline{\mathcal{A}}_{0}(m ;[k]-J)=\{0\} \sqcup \mathcal{A}_{0}(m ;[k]-J), \quad \overline{\mathcal{A}}_{1}(I, J)=\{0\} \sqcup \mathcal{A}_{1}(I, J) .
\end{gathered}
$$

Since $\lambda^{2}=0$, by (3-29),

$$
\begin{aligned}
\mathcal{C}_{1, k}^{m, J}(\bar{\partial})= & \frac{1}{m !}\left(\langle\mu\rangle_{1,[m] \sqcup J} \sum_{p=1}^{n}\left\langle c_{1}\left(\widetilde{\mathbb{E}}^{*}\right)^{p-1} \mathrm{ev}_{0}^{*} c_{n-p}(T X),\left[\widetilde{\mathfrak{M}}_{(m,[k]-J)}\right]\right\rangle\right. \\
& \left.-\langle\lambda ; \mu\rangle_{1,[m] \sqcup J} \sum_{p=1}^{n-1}\left\langle c_{1}\left(\widetilde{\mathbb{E}}^{*}\right)^{p-1} \operatorname{ev}_{0}^{*} c_{n-1-p}(T X),\left[\widetilde{\mathfrak{M}}_{(m,[k]-J)}\right]\right\rangle\right) .
\end{aligned}
$$


For each $\varrho \in \overline{\mathcal{A}}_{0}(m ;[k]-J)$, let $\bar{\pi}_{\varrho}: \widetilde{\mathfrak{M}}_{(m,[k]-J)} \longrightarrow \widetilde{\mathfrak{M}}_{(m,[k]-J)}^{\varrho}$ be the blowdown map. By (3-27),

$$
c_{1}(\widetilde{\mathbb{E}})=\bar{\pi}_{0}^{*} c_{1}\left(\gamma_{(m,[k]-J)}\right)+\sum_{\varrho \in \mathcal{A}_{0}(m ;[k-J)} \bar{\pi}_{\varrho}^{*} \widetilde{\mathfrak{M}}_{\varrho}^{\varrho} .
$$

Thus, we find that

$$
\begin{aligned}
c_{1}(\widetilde{\mathbb{E}})^{p-1}= & \bar{\pi}_{0}^{*} c_{1}\left(\gamma_{(m,[k]-J)}\right)^{p-1} \\
& +\sum_{\varrho \in \mathcal{A}_{0}(m ;[k]-J) q=1} \sum_{\varrho^{\prime}<\varrho}^{p-1}\left(\lambda+\sum_{\varrho^{\prime}} \bar{\pi}^{*} \widetilde{\mathfrak{M}}_{\varrho^{\prime}}\right)^{p-1-q}\left(\lambda+\sum_{\varrho^{\prime} \leq \varrho} \bar{\pi}_{\varrho^{\prime}}^{*} \widetilde{\mathfrak{M}}_{\varrho}^{\varrho}\right)^{q-1} \bar{\pi}_{\varrho^{\prime}}^{*} \widetilde{\mathfrak{M}}_{\varrho}^{\varrho} \\
& =\bar{\pi}_{0}^{*} c_{1}\left(\gamma_{(m,[k]-J)}\right)^{p-1} \\
& +\sum_{\varrho \in \mathcal{A}_{0}(m ;[k]-J)} \bar{\pi}_{\varrho}^{*}\left(\sum_{q=1}^{p-1}\left(\tilde{\pi}_{\varrho}^{*} c_{1}\left(\mathbb{E}_{\varrho-1}\right)^{p-1-q} c_{1}\left(\mathbb{E}_{\varrho}\right)^{q-1}\right) \cap \widetilde{\mathfrak{M}}_{\varrho}^{\varrho}\right) .
\end{aligned}
$$

Note that for every $\varrho \in \overline{\mathcal{A}}_{0}(m ;[k]-J)$

$$
\begin{aligned}
\left\langle c_{1}\left(\gamma_{\left(m_{B}(\varrho), J_{B}(\varrho)\right)}^{*}\right)^{q-1} \mathrm{ev}_{0}^{*} c_{r}(T X),\left[\widetilde{\mathfrak{M}}_{\left(m_{B}(\varrho), J_{B}(\varrho)\right)}^{0}\right]\right\rangle \\
=\left\langle\eta_{q-m_{B}(\varrho)} \operatorname{ev}_{0}^{*} c_{r}(T X),\left[\overline{\mathfrak{M}}_{\left(m_{B}(\varrho), J_{B}(\varrho)\right)}\right]\right\rangle \\
=m !\left|G_{\varrho}\right| \mathrm{GW}_{\left(m_{B}(\varrho), J_{B}(\varrho)\right)}^{\beta}\left(\eta_{q-m_{B}(\varrho)}, c_{r}(T X) ; \mu\right),
\end{aligned}
$$

with $\left(m_{B}(0), J_{B}(0)\right) \equiv(m ;[k]-J)$ and $\left|G_{0}\right| \equiv 1$.

Thus, by (3-28) and (3-30),

$$
\begin{aligned}
\mathcal{C}_{1, k}^{m, J}(\bar{\partial})= & \sum_{\rho \in \overline{\mathcal{A}}_{0}(m ;[k]-J)} \sum_{p=1}^{p=n} \sum_{q=1}^{q=p}\left\{(-1)^{p-q}\left\langle\tilde{\psi}^{p-1-q} ; \mu\right\rangle_{\left(0, \varrho_{P}\right)}\right. \\
& \times\left(\langle\mu\rangle_{1,[m] \sqcup J} \mathrm{GW}_{\left(m_{B}(\varrho), J_{B}(\varrho)\right)}^{\beta}\left(\eta_{q-m_{B}(\varrho)}, c_{n-p}(T X) ; \mu\right)\right. \\
& \left.\left.-\langle\lambda ; \mu\rangle_{1,[m] \sqcup J} \mathrm{GW}_{\left(m_{B}(\varrho), J_{B}(\varrho)\right)}^{\beta}\left(\eta_{q-m_{B}(\varrho)}, c_{n-1-p}(T X) ; \mu\right)\right)\right\},
\end{aligned}
$$

where we set

$$
\left\langle\tilde{\psi}^{r} ; \mu\right\rangle_{\left(0,0_{P}\right)}= \begin{cases}1, & \text { if } r=-1 \\ 0, & \text { otherwise }\end{cases}
$$


Most terms in (3-31) vanish for dimensional reasons. By $(2-6), \mathcal{C}_{1, k}^{m, J}(\bar{\partial})$ is thus given by

$$
\begin{aligned}
& \sum_{m^{*}=m}^{\infty} \sum_{J \subset J^{*} \subset[k]} \sum_{\substack{\rho \in \overline{\mathcal{A}}_{1}\left(\left[m^{*}\right], J^{*}\right) \\
\left|I_{P}(\rho)\right|+|\mathcal{N}(\rho)|=m \\
J_{P}(\rho)=J}} \\
& \left(\sum _ { q = 0 } ^ { n - m ^ { * } } \left\{(-1)^{n-m^{*}-d_{m^{*}, J^{*}}(\mu)} \mathrm{GW}_{\left(m^{*}, J^{*}\right)}^{\beta}\left(\eta_{q}, c_{d_{m^{*}, J^{*}}(\mu)-q} ; \mu\right)\right.\right. \\
& \times\left(\langle\mu\rangle_{1,[m] \sqcup J}\left\langle\widetilde{\psi}^{n-m^{*}-d_{m^{*}, J^{*}}(\mu)-1} ; \mu\right\rangle_{\left(0, \varrho_{B}(\rho)\right)}\right. \\
& \left.\left.\left.+\langle\lambda ; \mu\rangle_{1,[m] \sqcup J}\left\langle\tilde{\psi}^{n-m^{*}-d_{m^{*}, J^{*}}(\mu)-2} ; \mu\right\rangle_{\left(0, \varrho_{B}(\rho)\right)}\right)\right\}\right) .
\end{aligned}
$$

Summing over all $(m, J)$ as required by (3-20) and using the last expression in (2-5), we obtain

$$
\begin{aligned}
& \mathrm{GW}_{1, k}^{\beta}(\mu)-\mathrm{GW}_{1, k}^{\beta ; 0}(\mu) \\
& =\sum_{m^{*}=1}^{\infty} \sum_{J^{*} \subset[k]}\left\{(-1)^{m^{*}+\left|J^{*}\right|-p_{J^{*}}(\mu)} \times\left(\sum_{q=0}^{d_{m^{*}, J^{*}}(\mu)} \mathrm{GW}_{\left(m^{*}, J^{*}\right)}^{\beta}\left(\eta_{q}, c_{d_{m^{*}, J^{*}}(\mu)-q} ; \mu\right)\right)\right. \\
& \times \sum_{\rho \in \overline{\mathcal{A}}_{1}\left(\left[m^{*}\right], J^{*}\right)}\left(\langle\mu\rangle_{1, I_{P}(\rho) \sqcup J_{P}(\rho) \sqcup \aleph(\rho)}\left\langle\tilde{\psi}^{m^{*}+\left|J^{*}\right|-p_{J^{*}}(\mu)-1} ; \mu\right\rangle_{\left(0, \varrho_{B}(\rho)\right)}\right. \\
& \left.\left.+\langle\lambda ; \mu\rangle_{1, I_{P}(\rho) \sqcup J_{P}(\rho) \sqcup \aleph(\rho)}\left\langle\tilde{\psi}^{m^{*}+\left|J^{*}\right|-p_{J^{*}}(\mu)-2} ; \mu\right\rangle_{\left(0, \varrho_{B}(\rho)\right)}\right)\right\} .
\end{aligned}
$$

Finally, Proposition 3.1 reduces the last expression to the statement of Theorem 1A.

Remark Since $\widetilde{\mathfrak{M}}_{(m,[k]-J)}^{0}$ is not a complex manifold, some care is needed in constructing its "complex" blowups. These are obtained by modifying normal neighborhoods to the strata of the blowup centers in the expected way. The information needed to specify the normal bundles to such strata is described in Subsection 2.4 of [15]. Similarly, (3-27) describes a twisting of line bundles, not of sheaves. In fact, we know a priori that $N\left(\widetilde{\mathcal{D}}_{0}\right)$ depends only on the topology of the situation:

(T1) the domain and target bundles of $\widetilde{\mathcal{D}}_{0}$;

(T2) the normal bundles to the strata of $\widetilde{\mathcal{D}}_{0}^{-1}(0)$;

(T3) the topological behavior of $\widetilde{\mathcal{D}}_{0}$ in the normal directions to the strata of $\widetilde{\mathcal{D}}_{0}^{-1}(0)$. 
By constructing a tree of Chern classes, as suggested above and similarly to Subsection 3.2 in [18], one can obtain a universal formula expressing $N\left(\widetilde{\mathcal{D}}_{0}\right)$ in terms of the Chern class of (T1) and (T2) evaluated on the closures of the strata of $\widetilde{\mathcal{D}}_{0}^{-1}(0)$, with the coefficients determined by (T3). If such a universal formula holds in the presence of additional geometry (eg in the complex category), it must hold in general. Thus, it is sufficient to obtain a formula for $N\left(\widetilde{\mathcal{D}}_{0}\right)$ assuming $\widetilde{\mathfrak{M}}_{(m,[k]-J)}^{0}$ is a complex manifold.

\section{References}

[1] M Bershadsky, S Cecotti, H Ooguri, C Vafa, Holomorphic anomalies in topological field theories, Nuclear Phys. B 405 (1993) 279-304 MR1240687

[2] P Candelas, X C de la Ossa, PS Green, L Parkes, A pair of Calabi-Yau manifolds as an exactly soluble superconformal theory, Nuclear Phys. B 359 (1991) 21-74 MR1115626

[3] K Fukaya, K Ono, Arnold conjecture and Gromov-Witten invariant, Topology 38 (1999) 933-1048 MR1688434

[4] A Gathmann, Gromov-Witten invariants of hypersurfaces, Habilitation thesis, Univ of Kaiserslautern (2003)

[5] K Hori, S Katz, A Klemm, R Pandharipande, R Thomas, C Vafa, R Vakil, E Zaslow, Mirror symmetry, Clay Math. Monographs 1, Amer. Math. Soc. (2003) MR2003030With a preface by Vafa

[6] A Klemm, R Pandharipande, Enumerative geometry of Calabi-Yau 4-folds, to appear in Comm. Math. Phys. arXiv:math.AG/0702189

[7] J Li, G Tian, Virtual moduli cycles and Gromov-Witten invariants of general symplectic manifolds, from: "Topics in symplectic 4-manifolds (Irvine, CA, 1996)", First Int. Press Lect. Ser. I, Int. Press, Cambridge, MA (1998) 47-83 MR1635695

[8] J Li, A Zinger, On the genus-one Gromov-Witten invariants of complete intersections arXiv:math/0507104

[9] D Maulik, R Pandharipande, New calculations in Gromov-Witten theory, to appear in PAMQ arXiv:math.AG/0601395

[10] D Maulik, R Pandharipande, A topological view of Gromov-Witten theory, Topology 45 (2006) 887-918 MR2248516

[11] R Pandharipande, Intersections of $\mathbf{Q}$-divisors on Kontsevich's moduli space $\bar{M}_{0, n}\left(\mathbf{P}^{r}, d\right)$ and enumerative geometry, Trans. Amer. Math. Soc. 351 (1999) 14811505 MR1407707

[12] R Vakil, A Zinger, A natural smooth compactification of the space of elliptic curves in projective space, Electron. Res. Announc. Amer. Math. Soc. 13 (2007) 53-59 MR2320682 
[13] R Vakil, A Zinger, A desingularization of the main component of the moduli space of genus-one stable maps into $\mathbb{P}^{n}$, Geom. Topol. 12 (2008) 1-95 MR2377245

[14] D Zagier, A Zinger, Some properties of hypergeometric series associated with mirror symmetry, from: "Modular Forms and String Duality", Fields Institute Communications 54, Amer. Math. Soc. arXiv:0710.0889 Papers from the workshop held at BIRS

[15] A Zinger, Reduced genus-one Gromov-Witten invariants arXiv:math/0507103

[16] A Zinger, The reduced genus-one Gromov-Witten invariants of Calabi-Yau hypersurfaces arXiv:0705.2397

[17] A Zinger, Enumeration of genus-two curves with a fixed complex structure in $\mathbb{P}^{2}$ and $\mathbb{P}^{3}$, J. Differential Geom. 65 (2003) 341-467 MR2064428

[18] A Zinger, Enumeration of one-nodal rational curves in projective spaces, Topology 43 (2004) 793-829 MR2061208

[19] A Zinger, Intersections of tautological classes on blowups of moduli spaces of genus-1 curves, Michigan Math. J. 55 (2007) 535-560 MR2372615

Department of Mathematics, SUNY Stony Brook

Stony Brook, NY 11794-3651, USA

azinger@math . sunysb.edu

http://www . math. sunysb.edu/ azinger

Proposed: Jim Bryan

Received: 3 August 2007

Seconded: Yasha Eliashberg, Gang Tian

Revised: 17 January 2008

Geometry $\&$ Topology, Volume 12 (2008) 